\title{
Parallel multilevel constructions for constant dimension codes 1
}

\author{
Shuangqing Liu, Yanxun Chang, Tao Feng \\ Department of Mathematics, Beijing Jiaotong University, Beijing 100044, P. R. China \\ 16118420@bjtu.edu.cn, yxchang@bjtu.edu.cn, tfeng@bjtu.edu.cn
}

\begin{abstract}
Constant dimension codes (CDCs), as special subspace codes, have received a lot of attention due to their application in random network coding. This paper introduces a family of new codes, called rank metric codes with given ranks (GRMCs), to generalize the parallel construction in [Xu and Chen, IEEE Trans. Inf. Theory, 64 (2018), 63156319] and the classic multilevel construction. A Singleton-like upper bound and a lower bound for GRMCs derived from Gabidulin codes are given. Via GRMCs, two effective constructions for CDCs are presented by combining the parallel construction and the multilevel construction. Many CDCs with larger size than the previously best known codes are given. The ratio between the new lower bound and the known upper bound for $(4 \delta, 2 \delta, 2 \delta)_{q}$-CDCs is calculated. It is greater than 0.99926 for any prime power $q$ and any $\delta \geq 3$.
\end{abstract}

Keywords: constant dimension code, rank-metric code, multilevel construction, parallel construction.

\section{Introduction}

Subspace codes, constant dimension codes in particular, have drawn significant attention due to the work by Kötter and Kschischang [20, where they presented an application of such codes for error correction in random network coding.

Let $\mathbb{F}_{q}$ be the finite field of order $q$, and $\mathbb{F}_{q}^{n}$ be the set of all vectors of length $n$ over $\mathbb{F}_{q} . \mathbb{F}_{q}^{n}$ is an $n$-dimensional vector space over $\mathbb{F}_{q}$. Given a nonnegative integer $k \leq n$, the set of all $k$-dimensional subspaces of $\mathbb{F}_{q}^{n}$ is called the Grassmannian $\mathcal{G}_{q}(n, k)$. The cardinality of $\mathcal{G}_{q}(n, k)$ is given by the $q$-ary Gaussian coefficient

$$
\left|\mathcal{G}_{q}(n, k)\right|=\left[\begin{array}{l}
n \\
k
\end{array}\right]_{q} \triangleq \prod_{i=0}^{k-1} \frac{q^{n-i}-1}{q^{k-i}-1} .
$$

For any two subspaces $\mathcal{U}, \mathcal{V} \in \mathcal{G}_{q}(n, k)$, their subspace distance is defined by

$$
d_{S}(\mathcal{U}, \mathcal{V}) \triangleq \operatorname{dim} \mathcal{U}+\operatorname{dim} \mathcal{V}-2 \operatorname{dim}(\mathcal{U} \cap \mathcal{V})=2(k-\operatorname{dim}(\mathcal{U} \cap \mathcal{V}))
$$

A subset $\mathcal{C}$ of the Grassmannian $\mathcal{G}_{q}(n, k)$ is called an $(n, d, k)_{q}$ constant-dimension code $(\mathrm{CDC})$, if $d_{S}(\mathcal{U}, \mathcal{V}) \geq d$ for all $\mathcal{U}, \mathcal{V} \in \mathcal{C}$ and $\mathcal{U} \neq \mathcal{V}$. Elements in $\mathcal{C}$ are called codewords. An $(n, d, k)_{q}$-CDC with $M$ codewords is written as an $(n, M, d, k)_{q}$-CDC. Given $n, d, k$ and $q$, denote by $A_{q}(n, d, k)$ the maximum number of codewords among all $(n, d, k)_{q}$-CDCs. An $(n, d, k)_{q}$-CDC with $A_{q}(n, d, k)$ codewords is said to be optimal.

Without loss of generality, assume that $n \geq 2 k$. This assumption can be made as a consequence of the fact $A_{q}(n, d, k)=A_{q}(n, d, n-k)$, which can be obtained by taking

\footnotetext{
${ }^{1}$ Supported by NSFC under Grant 11971053 (Y. Chang), and NSFC under Grant 11871095 (T. Feng).
} 
orthogonal complements of subspaces (cf. [30]). Furthermore, by (1.1), when $2 k<d$, any nonempty $(n, d, k)_{q}$-CDC consists of exactly one codeword. Therefore, we always assume that $n \geq 2 k \geq d$.

A $k$-dimensional subspace $\mathcal{U}$ of $\mathbb{F}_{q}^{n}$ can be represented by a $k \times n$ generator matrix $\boldsymbol{U}$ whose rows form a basis of $\mathcal{U}$. Let $\mathbb{F}_{q}^{k \times n}$ denote the set of all $k \times n$ matrices over $\mathbb{F}_{q}$. For $\mathcal{U}, \mathcal{V} \in \mathcal{G}_{q}(n, k)$, the subspace distance on $\mathcal{G}_{q}(n, k)$ is also given by

$$
d_{S}(\mathcal{U}, \mathcal{V})=2 \cdot \operatorname{rank}\left(\begin{array}{l}
\boldsymbol{U} \\
\boldsymbol{V}
\end{array}\right)-2 k
$$

where $\boldsymbol{U}, \boldsymbol{V} \in \mathbb{F}_{q}^{k \times n}$ are matrices such that $\mathcal{U}=\operatorname{rowspace}(\boldsymbol{U})$ and $\mathcal{V}=\operatorname{rowspace}(\boldsymbol{V})$. The two matrices are usually not unique.

By (1.2), in a CDC, the minimum subspace distance $d$ is even. If $d=0$ or 2 , then all $k$-dimensional subspaces of $\mathbb{F}_{q}^{n}$ constitute an optimal $(n, d, k)_{q}$-CDC. It follows that $d=4$ is the minimum nontrivial value.

\subsection{Lifted maximum rank distance codes}

To obtain optimal CDCs, Silva, Kschischang and Kötter 25] pointed out that lifted maximum rank distance (MRD) codes can result in asymptotically optimal CDCs, and can be decoded efficiently in the context of random linear network coding.

For a matrix $\boldsymbol{A} \in \mathbb{F}_{q}^{m \times n}$, the rank of $\boldsymbol{A}$ is denoted by $\operatorname{rank}(\boldsymbol{A})$. The set $\mathbb{F}_{q}^{m \times n}$ is an $\mathbb{F}_{q}$-vector space. The rank distance on $\mathbb{F}_{q}^{m \times n}$ is defined by

$$
d_{R}(\boldsymbol{A}, \boldsymbol{B})=\operatorname{rank}(\boldsymbol{A}-\boldsymbol{B}), \text { for } \boldsymbol{A}, \boldsymbol{B} \in \mathbb{F}_{q}^{m \times n} .
$$

An $[m \times n, k, \delta]_{q}$ rank-metric code $\mathcal{D}$ is a $k$-dimensional $\mathbb{F}_{q}$-linear subspace of $\mathbb{F}_{q}^{m \times n}$ with minimum rank distance

$$
\delta=\min _{\boldsymbol{A}, \boldsymbol{B} \in \mathcal{D}, \boldsymbol{A} \neq \boldsymbol{B}}\left\{d_{R}(\boldsymbol{A}, \boldsymbol{B})\right\}
$$

Clearly

$$
\delta=\min _{\boldsymbol{A} \in \mathcal{D}, \boldsymbol{A} \neq \mathbf{0}}\{\operatorname{rank}(\boldsymbol{A})\} .
$$

Elements in $\mathcal{D}$ are called codewords. The Singleton-like upper bound for rank-metric codes implies that

$$
k \leq \max \{m, n\}(\min \{m, n\}-\delta+1)
$$

holds for any $[m \times n, k, \delta]_{q}$ code. When the equality holds, $\mathcal{D}$ is called a linear maximum rank distance code, denoted by an $\operatorname{MRD}[m \times n, \delta]_{q}$ code. Linear MRD codes exists for all feasible parameters (cf. [4,10,21]).

Write $\boldsymbol{I}_{k}$ as the $k \times k$ identity matrix.

Proposition 1.1 (Lifted MRD codes, [25]) Let $n \geq 2 k$. The lifted $M R D$ code

$$
\mathcal{C}=\left\{\operatorname{rowspace}\left(\boldsymbol{I}_{k} \mid \boldsymbol{A}\right): \boldsymbol{A} \in \mathcal{D}\right\}
$$

is an $\left(n, q^{(n-k)(k-\delta+1)}, 2 \delta, k\right)_{q}-C D C$, where $\mathcal{D}$ is an $M R D[k \times(n-k), \delta]_{q}$ code. 
We outline the proof of Proposition 1.1 for later use. It suffices to check the subspace distance of $\mathcal{C}$. For any $\mathcal{U}, \mathcal{V} \in \mathcal{C}$ and $\mathcal{U} \neq \mathcal{V}$, where $\mathcal{U}=\operatorname{rowspace}\left(\boldsymbol{I}_{k} \mid \boldsymbol{A}\right)$ and $\mathcal{V}=$ rowspace $\left(\boldsymbol{I}_{k} \mid \boldsymbol{B}\right)$, we have

$$
\begin{aligned}
d_{S}(\mathcal{U}, \mathcal{V}) & =2 \cdot \operatorname{rank}\left(\begin{array}{cc}
\boldsymbol{I}_{k} & \boldsymbol{A} \\
\boldsymbol{I}_{k} & \boldsymbol{B}
\end{array}\right)-2 k=2 \cdot \operatorname{rank}\left(\begin{array}{cc}
\boldsymbol{I}_{k} & \boldsymbol{A} \\
\boldsymbol{O} & \boldsymbol{B}-\boldsymbol{A}
\end{array}\right)-2 k \\
& =2 \cdot \operatorname{rank}(\boldsymbol{B}-\boldsymbol{A}) \geq 2 \delta .
\end{aligned}
$$

Given $n, \delta, k$ and $q$, denote by $\bar{A}_{q}(n, 2 \delta, k)$ the maximum number of codewords among all $(n, 2 \delta, k)_{q}$-CDC containing a lifted MRD code $\left(n, q^{(n-k)(k-\delta+1)}, 2 \delta, k\right)_{q}$-CDC as a subset. Many constructions for CDCs with large number of codewords known in the literature (cf. [6,11,22,24,26,28]) produce codes containing a lifted MRD code. This motivates the study, initialized by Etzion and Silberstein [7, on determining the lower and upper bounds on $\bar{A}_{q}(n, d, k)$.

Etzion and Silberstein [6] presented a simple but effective construction, named the multilevel construction, which generalizes the lifted MRD codes. Trautmann and Rosenthal in [28] improved the multilevel construction by pending dots. Using the idea of pending dots and graph matchings, Etzion, Silberstein in [7] and Silberstein, Trautmann in 24] constructed large subspace codes in $\mathcal{G}_{q}(n, k)$ of minimum subspace distance $d=4$ or $2 k-2$. $\mathrm{Xu}$ and Chen [31] presented a new construction which can be also seen as a generalization of the lifted MRD codes. Heinlein [14] summarized the upper bounds of CDCs which contain lifted MRD codes as follows.

Theorem 1.2 [14, Theorem 1] For $n \geq 2 k$, let $\mathcal{C}$ be an $(n, 2 \delta, k)_{q}-C D C$ which contains a lifted MRD code.

(1) If $k<2 \delta$ and $n \geq 3 \delta$, then $\bar{A}_{q}(n, 2 \delta, k) \leq q^{(n-k)(k-\delta+1)}+A_{q}(n-k, 2(2 \delta-k), \delta)$. If additionally $k=\delta, n \equiv r(\bmod k), 0 \leq r<k$, and $\left[\begin{array}{l}r \\ 1\end{array}\right]_{q}<k$, or $(n, 2 \delta, k) \in$ $\{(6+3 l, 4+2 l, 3+l),(6 l, 4 l, 3 l) \mid l \geq 1\}$, then the bound can be achieved.

(2) If $k<2 \delta$ and $n<3 \delta$, then $\bar{A}_{q}(n, 2 \delta, k)=q^{(n-k)(k-\delta+1)}+1$.

(3) If $2 \delta \leq k<3 \delta$, then

$$
\begin{aligned}
\bar{A}_{q}(n, 2 \delta, k) \leq q^{(n-k)(k-\delta+1)} & +A_{q}(n-k, 6 \delta-2 k, 2 \delta) \\
& +q^{(k-2 \delta+1)(n-k-\delta)} \frac{\left[\begin{array}{c}
n-k \\
\delta
\end{array}\right]_{q}\left[\begin{array}{c}
k \\
2 \delta-1
\end{array}\right]_{q}}{\left[\begin{array}{c}
k-\delta \\
\delta-1
\end{array}\right]_{q}}
\end{aligned}
$$

For more information on constructions and bounds for subspace codes, the interested reader is refered to $[1,3,7,9,12,13,16,19,20,23,27,29,30]$.

\subsection{Our contribution}

This paper is devoted to constructing large constant dimension codes which contain a lifted MRD code as a subset. 
Section 2 generalizes a construction for CDCs in 31] by introducing a family of new codes, called rank metric codes with given ranks (GRMCs). This generalized construction is called a parallel construction (see Construction 2.5). We shall establish a Singleton-like upper bound (see Proposition 2.3) and a lower bound (see Proposition 2.4) for GRMCs by using Gabidulin codes. Very recently, Heinlein [15] also introduced a similar concept to GRMCs. He presented several lower bounds for GRMCs, but most focus on special parameters. Here our construction is for general parameters. Applying Construction 2.5 together with Proposition 2.4, we give a lower bound on $\bar{A}_{q}(n, 2 \delta, k)$ for any $n \geq 2 k>$ $2 \delta>0$.

Section 3 presents two effective constructions for CDCs (see Constructions 3.10 and 3.17) by combining the parallel construction and the classic multilevel construction. Constructions 3.10 shows that if a multilevel construction satisfies the weight of the first $n-k$ positions of every identifying vector is no less than $\delta$, then the multilevel construction can be combined with a parallel construction. Constructions 3.17 shows that if identifying vectors in a multilevel construction dissatisfy the condition in Constructions 3.10 , the multilevel construction is still possible to be combined with a parallel construction. In both construction, GRMCs play an important role.

In principle, people can always pick up suitable identifying vectors and then use the classic multilevel construction to construct optimal CDC. However, how to choose identifying vectors effectively is still an open and different problem. The combination of the parallel construction and the multilevel construction helps to weaken the requirement for identifying vectors and provides good constant dimension codes with large size. Applying Constructions 3.10 and 3.17, we establish new lower bounds for CDCs (see Theorems 3.12, 3.14, 3.16 and Corollary 3.18). Many CDCs with larger size than the previously best known codes in [16] are given (see Appendix B). We also calculate the ratio between our lower bound and the known upper bound for $(4 \delta, 2 \delta, 2 \delta)_{q}$-CDCs. It is greater than 0.99926 for any prime power $q$ and any $\delta \geq 3$ (see Remark 3.201).

\section{Parallel construction}

In [31], $\mathrm{Xu}$ and Chen presented an interesting construction to establish new lower bounds for $A_{q}(2 k, 2 \delta, k)$, where $k \geq 2 \delta$.

Theorem 2.1 31, Theorem 3 and Corollary 4] For any positive integers $k$ and $\delta$ such that $k \geq 2 \delta$,

$$
A_{q}(2 k, 2 \delta, k) \geq q^{k(k-\delta+1)}+\sum_{i=\delta}^{k-\delta} A_{i},
$$

where $A_{i}$ denotes the number of codewords with rank $i$ in an $M R D[k \times k, \delta]_{q}$ code.

Theorem 2.1 relies on the use of the rank distribution of an MRD code.

Theorem 2.2 (Rank distribution [4, 10]) Let $m \geq n$. Let $\mathcal{D}$ be an $M R D[m \times n, \delta]_{q}$ code, and $A_{i}=|\{M \in \mathcal{D}: \operatorname{rank}(M)=i\}|$ for $0 \leq i \leq n$. Its rank distribution is given by $A_{0}=1, A_{i}=0$ for $1 \leq i \leq \delta-1$, and

$$
A_{\delta+i}=\left[\begin{array}{c}
n \\
\delta+i
\end{array}\right]_{q} \sum_{j=0}^{i}(-1)^{j-i}\left[\begin{array}{l}
\delta+i \\
i-j
\end{array}\right]_{q} q^{\left(\begin{array}{c}
i-j \\
2
\end{array}\right)}\left(q^{m(j+1)}-1\right)
$$

for $0 \leq i \leq n-\delta$. 
In this section, we shall generalize Theorem 2.1 by introducing the concept of rank metric codes with given ranks (GRMCs), which can be seen as a generalization of constant-rank codes. Constant-rank codes have been discussed systematically in [11].

We remark that very recently, based on Theorem 2.1, Chen et.al [3] and Heinlein [15] generalized linkage constructions to establish some lower bounds of CDCs independently.

\subsection{Rank metric codes with given ranks}

Let $K \subseteq\{0,1, \ldots, n\}$ and $\delta$ be a positive integer. We say $\mathcal{D} \subseteq \mathbb{F}_{q}^{m \times n}$ is an $(m \times n, \delta, K)_{q}$ rank metric code with given ranks (GRMC) if it satisfies

(1) $\operatorname{rank}(\boldsymbol{D}) \in K$ for any $\boldsymbol{D} \in \mathcal{D}$;

(2) $d_{R}\left(\boldsymbol{D}_{1}, \boldsymbol{D}_{2}\right)=\operatorname{rank}\left(\boldsymbol{D}_{1}-\boldsymbol{D}_{2}\right) \geq \delta$ for any $\boldsymbol{D}_{1}, \boldsymbol{D}_{2} \in \mathcal{D}$ and $\boldsymbol{D}_{1} \neq \boldsymbol{D}_{2}$.

If $|\mathcal{D}|=M$, then it is often written as an $(m \times n, M, \delta, K)_{q}$-GRMC. Given $m, n, K$ and $\delta$, denote by $A_{q}^{R}(m \times n, \delta, K)$ the maximum number of codewords among all $(m \times n, \delta, K)_{q^{-}}$ GRMCs.

When $K=\{0,1, \ldots, n\}$, a GRMC is just a usual rank-metric code (not necessarily linear). When $K=\{t\}$ for $0 \leq t \leq n$, a GRMC is often called a constant-rank code (cf. [11]). Consequently, when $m \geq n$,

$$
\max _{t \in K} A_{q}^{R}(m \times n, \delta, t) \leq A_{q}^{R}(m \times n, \delta, K) \leq q^{m(n-\delta+1)} .
$$

Usually, $K$ is selected as a set of consecutive integers. Let $\left[t_{1}, t_{2}\right]$ denote the set of integers $k$ such that $t_{1} \leq k \leq t_{2}$.

Proposition 2.3 (Singleton-like upper bound) For all $0 \leq i, j \leq \min \left\{\delta-1, t_{1}\right\}$,

$$
A_{q}^{R}\left(m \times n, \delta,\left[t_{1}, t_{2}\right]\right) \leq A_{q}^{R}\left((m-i) \times(n-j), \delta-l,\left[t_{1}-l, t\right]\right),
$$

where $l=\max \{i, j\}$ and $t=\min \left\{m-i, n-j, t_{2}\right\}$.

Proof Let $\mathcal{D}$ be any $\left(m \times n, M, \delta,\left[t_{1}, t_{2}\right]\right)_{q}$-GRMC. For any $0 \leq i, j \leq \min \left\{\delta-1, t_{1}\right\}$, the $\mathcal{D}_{i j}$ is obtained by removing the same $i$ rows and $j$ columns from every codeword in $\mathcal{D}$. Then $\mathcal{D}_{i j}$ is an $\left((m-i) \times(n-j), M, \delta-l,\left[t_{1}-l, t\right]\right)_{q}$-GRMC.

When $t_{1}=t_{2}$, Proposition 2.3 provides an upper bound for constant-rank codes (see also [11, Proposition 7]).

We remark that very recently, Heinlein [15] also introduced the concept of GRMCs for the case $K=\left[0, t_{2}\right]$. He established a similar upper bound for GRMCs to that in Proposition 2.3. He also presented several lower bounds for GRMCs, but most focus on special parameters. Here we shall give a general construction on GRMCs.

To construct GRMCs, we need a special class of MRD codes, named Gabidulin codes. Let $\boldsymbol{\beta}=\left(\beta_{0}, \beta_{1}, \ldots, \beta_{m-1}\right)$ be an ordered basis of $\mathbb{F}_{q^{m}}$ over $\mathbb{F}_{q}$. There is a natural bijective map $\Psi_{m}$ from $\mathbb{F}_{q^{m}}^{n}$ to $\mathbb{F}_{q}^{m \times n}$ as follows:

$$
\begin{gathered}
\Psi_{m}: \mathbb{F}_{q^{m}}^{n} \longrightarrow \mathbb{F}_{q}^{m \times n} \\
\mathbf{a}=\left(a_{0}, a_{1}, \ldots, a_{n-1}\right) \longmapsto \boldsymbol{A},
\end{gathered}
$$


where $\boldsymbol{A}=\Psi_{m}(\mathbf{a}) \in \mathbb{F}_{q}^{m \times n}$ is defined such that

$$
a_{j}=\sum_{i=0}^{m-1} A_{i, j} \beta_{i}
$$

for any $j \in[n]$. The map $\Psi_{m}$ will be used to facilitate switching between a vector in $\mathbb{F}_{q^{m}}$ and its matrix representation over $\mathbb{F}_{q}$. In the sequel, we use both representations, depending on what is more convenient in the context.

For any positive integer $i$ and any $a \in \mathbb{F}_{q^{m}}$, set $a^{[i]} \triangleq a^{q^{i}}$. Let $m \geq n$ and $\delta$ be a positive integer. A Gabidulin code $\mathcal{G}[m \times n, \delta]_{q}$ is an $\operatorname{MRD}[m \times n, \delta]_{q}$ code whose generator matrix $\boldsymbol{G}$ in vector representation is

$$
\boldsymbol{G}=\left(\begin{array}{cccc}
g_{0} & g_{1} & \cdots & g_{n-1} \\
g_{0}^{[1]} & g_{1}^{[1]} & \cdots & g_{n-1}^{[1]} \\
\vdots & \vdots & \ddots & \vdots \\
g_{0}^{[n-\delta]} & g_{1}^{[n-\delta]} & \cdots & g_{n-1}^{[n-\delta]}
\end{array}\right)
$$

where $g_{0}, g_{1}, \ldots, g_{n-1} \in \mathbb{F}_{q^{m}}$ are linearly independent over $\mathbb{F}_{q}$ (see [10]). Then $\mathcal{G}[m \times n, \delta]_{q}$ can be written as $\left\{\boldsymbol{u} G: \boldsymbol{u} \in \mathbb{F}_{q^{m}}^{n-\delta+1}\right\}$.

Proposition 2.4 (Lower bound) Let $m \geq n$ and $1 \leq \delta \leq n$. Let $t_{1}$ be a nonnegative integer and $t_{2}$ be a positive integer such that $t_{1} \leq t_{2} \leq n$. Then

$$
A_{q}^{R}\left(m \times n, \delta,\left[t_{1}, t_{2}\right]\right) \geq \begin{cases}\sum_{i=t_{1}}^{t_{2}} A_{i}(\delta), & t_{2} \geq \delta ; \\ \max _{\max \left\{1, t_{1}\right\} \leq a<\delta}\left\{\left\lceil\frac{\sum_{i=\max \left\{1, t_{1}\right\}}^{t_{2}} A_{i}(a)}{q^{m(\delta-a)}-1}\right\rceil\right\}, & t_{2}<\delta,\end{cases}
$$

where $A_{i}(x)$ denotes the number of codewords with rank $i$ in an $M R D[m \times n, x]_{q}$ code.

Proof When $t_{2} \geq \delta$, all codewords with ranks from $\left[t_{1}, t_{2}\right]$ in an $\operatorname{MRD}[m \times n, \delta]_{q}$ code form an $\left(m \times n, \delta,\left[t_{1}, t_{2}\right]\right)_{q}$-GRMC with $\sum_{i=t_{1}}^{t_{2}} A_{i}(\delta)$ codewords. Thus $A_{q}^{R}(m \times$ $\left.n, \delta,\left[t_{1}, t_{2}\right]\right) \geq \sum_{i=t_{1}}^{t_{2}} A_{i}(\delta)$ for any $t_{2} \geq \delta$.

When $t_{2}<\delta$, take any integer $a$ such that $\max \left\{1, t_{1}\right\} \leq a<\delta$. Let $\mathcal{D}_{1}$ be a $\mathcal{G}[m \times n, \delta]_{q}$ code whose generator matrix $\boldsymbol{G}_{1}$ in vector representation is

$$
\boldsymbol{G}_{1}=\left(\begin{array}{cccc}
g_{0} & g_{1} & \cdots & g_{n-1} \\
g_{0}^{[1]} & g_{1}^{[1]} & \cdots & g_{n-1}^{[1]} \\
\vdots & \vdots & \ddots & \vdots \\
g_{0}^{[n-\delta]} & g_{1}^{[n-\delta]} & \cdots & g_{n-1}^{[n-\delta]}
\end{array}\right)
$$

where $g_{0}, g_{1}, \ldots, g_{n-1} \in \mathbb{F}_{q^{m}}$ are linearly independent over $\mathbb{F}_{q}$. Let $\mathcal{D}_{2}$ be a $\mathcal{G}[m \times n, n-$ $\delta+a+1]_{q}$ code whose generator matrix $\boldsymbol{G}_{2}$ in vector representation is

$$
\boldsymbol{G}_{2}=\left(\begin{array}{cccc}
g_{0}^{[n-\delta+1]} & g_{1}^{[n-\delta+1]} & \ldots & g_{n-1}^{[n-\delta+1]} \\
g_{0}^{[n-\delta+2]} & g_{1}^{[n-\delta+2]} & \cdots & g_{n-1}^{[n-\delta+2]} \\
\vdots & \vdots & \ddots & \vdots \\
g_{0}^{[n-a]} & g_{1}^{[n-a]} & \cdots & g_{n-1}^{[n-a]}
\end{array}\right) .
$$


Note that $g_{0}^{[n-\delta+1]}, g_{1}^{[n-\delta+1]}, \cdots, g_{n-1}^{[n-\delta+1]}$ are also linearly independent over $\mathbb{F}_{q}$. Then

$$
\bigcup_{\boldsymbol{D}_{2} \in \mathcal{D}_{2}} \bigcup_{\boldsymbol{D}_{1} \in \mathcal{D}_{1}}\left(\boldsymbol{D}_{2}+\boldsymbol{D}_{1}\right)
$$

is a $\mathcal{G}[m \times n, a]_{q}$ code whose generator matrix is $\left(\begin{array}{c}\boldsymbol{G}_{1} \\ \boldsymbol{G}_{2}\end{array}\right)$. For any $\boldsymbol{D}_{2} \in \mathcal{D}_{2} \backslash\left\{\boldsymbol{O}_{m \times n}\right\}$, where $\boldsymbol{O}_{m \times n}$ is the $m \times n$ zero matrix, set

$$
\mathcal{S}_{\boldsymbol{D}_{2}}=\left\{\boldsymbol{D} \in \bigcup_{\boldsymbol{D}_{1} \in \mathcal{D}_{1}}\left(\boldsymbol{D}_{2}+\boldsymbol{D}_{1}\right) \mid \operatorname{rank}(\boldsymbol{D}) \in\left[t_{1}, t_{2}\right]\right\} .
$$

For any $\boldsymbol{D}_{1}, \boldsymbol{D}_{1}^{\prime} \in \mathcal{D}_{1}$ and $\boldsymbol{D}_{1} \neq \boldsymbol{D}_{1}^{\prime}$, we have $\operatorname{rank}\left(\left(\boldsymbol{D}_{2}+\boldsymbol{D}_{1}\right)-\left(\boldsymbol{D}_{2}+\boldsymbol{D}_{1}^{\prime}\right)\right) \geq \delta$. It follows that $\mathcal{S}_{\boldsymbol{D}_{2}}$ forms an $\left(m \times n, M_{\boldsymbol{D}_{2}}, \delta,\left[t_{1}, t_{2}\right]\right)_{q}$-GRMC, where by the pigeonhole principle,

$$
M_{\boldsymbol{D}_{2}} \geq\left\lceil\frac{\sum_{i=\max \left\{1, t_{1}\right\}}^{t_{2}} A_{i}(a)}{\left|\mathcal{D}_{2}\right|-1}\right\rceil=\left\lceil\frac{\sum_{i=\max \left\{1, t_{1}\right\}}^{t_{2}} A_{i}(a)}{q^{m(\delta-a)}-1}\right\rceil .
$$

Note that due to $\delta>t_{2}$, if $t_{1}>0$, then there is no codeword in $\mathcal{D}_{1}$ with rank $i \in\left[t_{1}, t_{2}\right]$. Therefore, $A_{q}^{R}\left(m \times n, \delta,\left[t_{1}, t_{2}\right]\right) \geq \max _{\max \left\{1, t_{1}\right\} \leq a<\delta}\left\{\left\lceil\frac{\sum_{i=\max \left\{1, t_{1}\right\}}^{t_{2}} A_{i}(a)}{q^{m(\delta-a)}-1}\right\rceil\right\}$.

\subsection{Generalization of Theorem 2.1}

Construction 2.5 (Parallel construction) Let $n \geq 2 k \geq 2 \delta$. If there exists a $(k \times$ $(n-k), M, \delta,[0, k-\delta])_{q}-G R M C$, then there exists an $\left(n, q^{(n-k)(k-\delta+1)}+M, 2 \delta, k\right)_{q}-C D C$, which contains a lifted MRD code $\left(n, q^{(n-k)(k-\delta+1)}, 2 \delta, k\right)_{q}-C D C$ as a subset.

Proof Let $\mathcal{D}_{1}$ be an $\operatorname{MRD}[k \times(n-k), \delta]_{q}$ code and $\mathcal{D}_{2}$ be a $(k \times(n-k), M, \delta,[0, k-\delta])_{q^{-}}$ GRMC. Let $\mathcal{C}_{1}=\left\{\operatorname{rowspace}\left(\boldsymbol{I}_{k} \mid \boldsymbol{A}\right): \boldsymbol{A} \in \mathcal{D}_{1}\right\}$ and $\mathcal{C}_{2}=\left\{\operatorname{rowspace}\left(\boldsymbol{B} \mid \boldsymbol{I}_{k}\right): \boldsymbol{B} \in \mathcal{D}_{2}\right\}$. Then $\mathcal{C}=\mathcal{C}_{1} \cup \mathcal{C}_{2}$ forms an $\left(n, q^{(n-k)(k-\delta+1)}+M, 2 \delta, k\right)_{q}$-CDC.

It suffices to check the subspace distance of $\mathcal{C}$. For any $\mathcal{U}=\operatorname{rowspace}\left(\boldsymbol{I}_{k} \mid \boldsymbol{A}\right) \in \mathcal{C}_{1}$ and $\mathcal{V}=\operatorname{rowspace}\left(\boldsymbol{B} \mid \boldsymbol{I}_{k}\right) \in \mathcal{C}_{2}$, where $\boldsymbol{A}=(\underbrace{\boldsymbol{A}_{1}}_{n-2 k} \mid \underbrace{\boldsymbol{A}_{2}}_{k})$ and $\boldsymbol{B}=(\underbrace{\boldsymbol{B}_{1}}_{k} \mid \underbrace{\boldsymbol{B}_{2}}_{n-2 k})$, we have

$$
\begin{aligned}
d_{S}(\mathcal{U}, \mathcal{V}) & =2 \cdot \operatorname{rank}\left(\begin{array}{ccc}
\boldsymbol{I}_{k} & \boldsymbol{A}_{1} & \boldsymbol{A}_{2} \\
\boldsymbol{B}_{1} & \boldsymbol{B}_{2} & \boldsymbol{I}_{k}
\end{array}\right)-2 k \\
& =2 \cdot \operatorname{rank}\left(\begin{array}{ccc}
\boldsymbol{I}_{k} & \boldsymbol{A}_{1} & \boldsymbol{A}_{2} \\
\boldsymbol{O} & \boldsymbol{B}_{2}-\boldsymbol{B}_{1} \boldsymbol{A}_{1} & \boldsymbol{I}_{k}-\boldsymbol{B}_{1} \boldsymbol{A}_{2}
\end{array}\right)-2 k \\
& =2 \cdot \operatorname{rank}\left(\boldsymbol{B}_{2}-\boldsymbol{B}_{1} \boldsymbol{A}_{1} \mid \boldsymbol{I}_{k}-\boldsymbol{B}_{1} \boldsymbol{A}_{2}\right) \\
& \geq 2 \cdot \operatorname{rank}\left(\boldsymbol{I}_{k}-\boldsymbol{B}_{1} \boldsymbol{A}_{2}\right) \\
& \geq 2 \cdot \operatorname{rank} \cdot\left(\boldsymbol{I}_{k}-\boldsymbol{B}_{1} \boldsymbol{A}_{2}+\boldsymbol{B}_{1} \boldsymbol{A}_{2}\right)-2 \cdot \operatorname{rank}\left(\boldsymbol{B}_{1} \boldsymbol{A}_{2}\right) \\
& =2 k-2 \cdot \operatorname{rank}\left(\boldsymbol{B}_{1} \boldsymbol{A}_{2}\right) \\
& \geq 2 k-2 \cdot \operatorname{rank}\left(\boldsymbol{B}_{1}\right) \geq 2 k-2 \cdot \operatorname{rank}(\boldsymbol{B}) \geq 2 \delta .
\end{aligned}
$$

Given $i \in\{1,2\}$, for any $\mathcal{U}, \mathcal{V} \in \mathcal{C}_{i}$ and $\mathcal{U} \neq \mathcal{V}$, we have $d_{S}(\mathcal{U}, \mathcal{V}) \geq 2 \delta$ by the proof of Proposition 1.1. 
Construction 2.5 comes from two parallel versions of lifted MRD codes, so it is named as a parallel construction. As a straightforward corollary of Construction 2.5, we have the following result.

Theorem 2.6 Let $n \geq 2 k \geq 2 \delta$. Then

$$
\bar{A}_{q}(n, 2 \delta, k) \geq q^{(n-k)(k-\delta+1)}+A_{q}^{R}(k \times(n-k), \delta,[0, k-\delta]) .
$$

Theorem 2.7 Let $n \geq 2 k>2 \delta>0$. Then

$$
\bar{A}_{q}(n, 2 \delta, k) \geq q^{(n-k)(k-\delta+1)}+ \begin{cases}\sum_{i=\delta}^{k-\delta} A_{i}(\delta)+1, & k \geq 2 \delta \\ \max _{1 \leq a<\delta}\left\{\left\lceil\frac{\sum_{i=1}^{k-\delta} A_{i}(a)}{q^{m(\delta-a)}-1}\right\rceil\right\}, & k<2 \delta,\end{cases}
$$

where $A_{i}(x)$ denotes the number of codewords with rank $i$ in an $M R D[m \times n, x]_{q}$ code.

Proof Apply Proposition 2.4 with $t_{1}=0$ and $t_{2}=k-\delta$. Then apply Theorem 2.6.

We remark that if $k \geq 2 \delta$, then Theorem 2.1 is a corollary of Theorem 2.7 by taking $n=2 k$.

\section{Parallel multilevel construction}

This section is devoted to presenting two effective constructions for constant dimension codes (Constructions 3.10 and 3.17) by combining the parallel construction shown in Section 2 and the multilevel construction introduced in [6]. First we give a revisit of the multilevel construction in [6].

\subsection{Preliminaries}

Etzion and Silberstein [6] presented the multilevel construction for constant dimension codes by establishing relations between subspace distances and Hamming distances or rank distances (see Lemmas 3.7 and 3.8, respectively, below). The multilevel construction is a generalization of Proposition 1.1 by introducing Ferrers diagram rank-metric codes.

\subsubsection{Ferrers diagram rank-metric codes}

Given positive integers $m$ and $n$, an $m \times n$ Ferrers diagram $\mathcal{F}$ is an $m \times n$ array of dots and empty cells such that all dots are shifted to the right of the diagram, the number of dots in each row is less than or equal to the number of dots in the previous row, and the first row has $n$ dots and the rightmost column has $m$ dots.

We always denote by $\gamma_{i}, 0 \leq i \leq n-1$, the number of dots in the $i$-th column of $\mathcal{F}$. Given positive integers $m$ and $n$, and $1 \leq \gamma_{0} \leq \gamma_{1} \leq \cdots \leq \gamma_{n-1}=m$, there exists a unique Ferrers diagram $\mathcal{F}$ of size $m \times n$ such that the $i$-th column of $\mathcal{F}$ has cardinality $\gamma_{i}$ for any $0 \leq i \leq n-1$. In this case we write $\mathcal{F}=\left[\gamma_{0}, \gamma_{1}, \ldots, \gamma_{n-1}\right]$.

Example 3.1 Let $\mathcal{F}=[2,3,4,5]$, where 


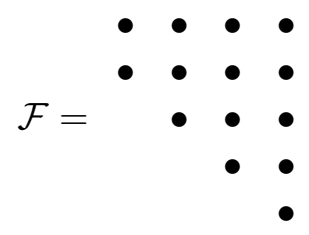

be a $5 \times 4$ Ferrers diagram.

For a given $m \times n$ Ferrers diagram $\mathcal{F}$, an $[\mathcal{F}, k, \delta]_{q}$ Ferrers diagram rank-metric code (FDRMC), briefly an $[\mathcal{F}, k, \delta]_{q}$ code, is an $[m \times n, k, \delta]_{q}$ rank-metric codes in which for each $m \times n$ matrix, all entries not in $\mathcal{F}$ are zero. If $\mathcal{F}$ is a full $m \times n$ diagram with $m n$ dots, then its corresponding FDRM code is just a classical rank-metric code. For a Ferrers diagram $\mathcal{F}$ of size $m \times n$, one can transpose it to obtain a Ferrers diagram $\mathcal{F}^{t}$ of size $n \times m$. If there exists an $[\mathcal{F}, k, \delta]_{q}$ code, then so does an $\left[\mathcal{F}^{t}, k, \delta\right]_{q}$ code. Etzion and Silberstein [6] established a Singleton-like upper bound on FDRMCs.

Lemma 3.2 [6. Theorem 1] Let $\delta$ be a positive integer. Let $v_{i}, 0 \leq i \leq \delta-1$, be the number of dots in a Ferrers diagram $\mathcal{F}$ which are not contained in the first $i$ rows and the rightmost $\delta-1-i$ columns. Then for any $[\mathcal{F}, k, \delta]_{q} \operatorname{code}, k \leq \min _{i \in\{0,1, \ldots, \delta-1\}} v_{i}$.

An FDRMC attaining the upper bound in Lemma 3.2 is called optimal. Constructions for optimal FDRMCs can be found in $[2,5,6,13,17,18,24,32$. We here only quote several known constructions for late use.

Theorem 3.3 [5, Theorem 3] Assume $\mathcal{F}$ is an $m \times n(m \geq n)$ Ferrers diagram and each of the rightmost $\delta-1$ columns of $\mathcal{F}$ has at least $n$ dots. Then there exists an optimal $\left[\mathcal{F}, \sum_{i=0}^{n-\delta} \gamma_{i}, \delta\right]_{q}$ code for any prime power $q$.

Theorem 3.4 [5, Theorem 9] Let $\mathcal{F}_{i}$ for $i=1,2$ be an $m_{i} \times n_{i}$ Ferrers diagram, and $\mathcal{C}_{i}$ be an $\left[\mathcal{F}_{i}, k, \delta_{i}\right]_{q}$ code. Let $\mathcal{F}_{3}$ be an $m_{3} \times n_{3}$ full Ferrers diagram with $m_{3} n_{3}$ dots, where $m_{3} \geq m_{1}$ and $n_{3} \geq n_{2}$. Let

$$
\mathcal{F}=\left(\begin{array}{cc}
\mathcal{F}_{1} & \mathcal{F}_{3} \\
& \mathcal{F}_{2}
\end{array}\right)
$$

be an $m \times n$ Ferrers diagram $\mathcal{F}$, where $m=m_{2}+m_{3}$ and $n=n_{1}+n_{3}$. Then there exists an $\left[\mathcal{F}, k, \delta_{1}+\delta_{2}\right]_{q}$ code $\mathcal{D}$ such that for any $\boldsymbol{D} \in \mathcal{D},\left.\boldsymbol{D}\right|_{\mathcal{F}_{3}}=\boldsymbol{O}$, where $\left.\boldsymbol{D}\right|_{\mathcal{F}_{3}}$ denotes the restriction of $\boldsymbol{D}$ in $\mathcal{F}_{3}$ and $\boldsymbol{O}$ is an $m_{3} \times n_{3}$ zero matrix.

The following theorem is a variation of Theorem 10 in [5].

Theorem 3.5 Let $\mathcal{F}_{12}=\left[\gamma_{0}, \gamma_{1}, \ldots, \gamma_{n_{1}-1}, \gamma_{n_{1}}, \ldots, \gamma_{n_{1}+n_{2}-1}\right]$ be a $\gamma_{n_{1}+n_{2}-1} \times\left(n_{1}+n_{2}\right)$ Ferrers diagram, which induces a $\gamma_{n_{1}-1} \times n_{1}$ Ferrers diagram $\mathcal{F}_{1}=\left[\gamma_{0}, \gamma_{1}, \ldots, \gamma_{n_{1}-1}\right]$ and a $\gamma_{n_{1}+n_{2}-1} \times n_{2}$ Ferrers diagram $\mathcal{F}_{2}=\left[\gamma_{n_{1}}, \gamma_{n_{1}+1}, \ldots, \gamma_{n_{1}+n_{2}-1}\right]$. Let $\mathcal{F}_{3}$ be a $\gamma_{n_{1}-1} \times$ $\gamma_{n_{1}+n_{2}-1}$ full Ferrers diagram with $\gamma_{n_{1}-1} \gamma_{n_{1}+n_{2}-1}$ dots. If there exists an $\left[\mathcal{F}_{12}, k, \delta\right]_{q}$ code, then there exists an $[\mathcal{F}, k, \delta]_{q}$ code $\mathcal{D}$, where

$$
\mathcal{F}=\left(\begin{array}{cc}
\mathcal{F}_{1} & \mathcal{F}_{3} \\
& \mathcal{F}_{2}^{t}
\end{array}\right)
$$

satisfying that for any codeword $\boldsymbol{D} \in \mathcal{D},\left.\boldsymbol{D}\right|_{\mathcal{F}_{3}}=\boldsymbol{O}$, where $\left.\boldsymbol{D}\right|_{\mathcal{F}_{3}}$ denotes the restriction of $\boldsymbol{D}$ in $\mathcal{F}_{3}$ and $\boldsymbol{O}$ is a $\gamma_{n_{1}-1} \times \gamma_{n_{1}+n_{2}-1}$ zero matrix. 
Proof Let $\mathcal{D}_{12}$ be the given $\left[\mathcal{F}_{12}, k, \delta\right]_{q}$ code. Set

$$
\mathcal{D}=\left\{\left(\begin{array}{cc}
\left.\boldsymbol{D}\right|_{\mathcal{F}_{1}} & \boldsymbol{O} \\
\boldsymbol{O} & \left.\boldsymbol{D}\right|_{\mathcal{F}_{2}^{t}}
\end{array}\right): \boldsymbol{D} \in \mathcal{D}_{12}\right\}
$$

where $\left.\boldsymbol{D}\right|_{\mathcal{F}_{1}}$ denotes the restriction of $\boldsymbol{D}$ in $\mathcal{F}_{1}$ and $\left.\boldsymbol{D}\right|_{\mathcal{F}_{2}^{t}}$ denotes the restriction of $\boldsymbol{D}$ in $\mathcal{F}_{2}^{t}$. Then $\mathcal{D}$ is an $[\mathcal{F}, k, \delta]_{q}$ code. One can easily verify the linearity and the dimension of $\mathcal{D}$. For any nonzero $\boldsymbol{D} \in \mathcal{D}_{12}$, we have

$$
\begin{aligned}
\operatorname{rank}\left(\begin{array}{cc}
\left.\boldsymbol{D}\right|_{\mathcal{F}_{1}} & \boldsymbol{O} \\
\boldsymbol{O} & \left.\boldsymbol{D}\right|_{\mathcal{F}_{2}^{t}}
\end{array}\right) & =\operatorname{rank}\left(\left.\boldsymbol{D}\right|_{\mathcal{F}_{1}}\right)+\operatorname{rank}\left(\left.\boldsymbol{D}\right|_{\mathcal{F}_{2}}\right) \\
& \geq \operatorname{rank}\left(\left.\boldsymbol{D}\right|_{\mathcal{F}_{1}}|\boldsymbol{D}|_{\mathcal{F}_{2}}\right)=\operatorname{rank}(\boldsymbol{D}) \geq \delta
\end{aligned}
$$

which proves the minimum rank distance of $\mathcal{D}$.

\subsubsection{Multilevel construction}

A matrix is said to be in row echelon form if each nonzero row has more leading zeros than the previous row. A matrix is in reduced row echelon form if (1) the leading coefficient of a row is always to the right of the leading coefficient of the previous row; (2) all leading coefficients are ones; (3) every leading coefficient is the only nonzero entry in its column.

A $k$-dimensional subspace $\mathcal{U}$ of $\mathbb{F}_{q}^{n}$ can be represented by a $k \times n$ generator matrix whose rows form a basis of $\mathcal{U}$. We usually represent a codeword of a constant dimension code by such a matrix. There is exactly one such matrix in reduced row echelon form and it will be denoted by $E(\mathcal{U})[6]$.

The identifying vector $\boldsymbol{v}(\mathcal{U})$ of a subspace $\mathcal{U} \in \mathcal{G}_{q}(n, k)$ is the binary vector of length $n$ and weight $k$ such that the $1^{\prime} s$ of $\boldsymbol{v}(\mathcal{U})$ are in the positions (columns) where $E(\mathcal{U})$ has its leading ones (of the rows). The echelon Ferrers form of a vector of length $n$ and weight $k, E F(\boldsymbol{v})$, is the matrix in reduced row echelon form with leading entries (of rows) in the columns indexed by the nonzero entries of $\boldsymbol{v}$ and $\bullet$ (called a dot) in all entries which do not have terminals zeros or ones.

Example 3.6 Consider the subspace $\mathcal{U} \in \mathcal{G}_{2}(7,3)$ with reduced row echelon form

$$
E(\mathcal{U})=\left(\begin{array}{lllllll}
1 & 0 & 0 & 0 & 1 & 1 & 0 \\
0 & 0 & 1 & 0 & 1 & 0 & 1 \\
0 & 0 & 0 & 1 & 0 & 1 & 1
\end{array}\right)
$$

Its identifying vector $\boldsymbol{v}(\mathcal{U})$ is 1011000. For the identifying vector $\boldsymbol{v}(\mathcal{U})=1011000$, its echelon Ferrers form $\operatorname{EF}(\boldsymbol{v}(\mathcal{U}))$ is the following $3 \times 7$ matrix:

$$
E F(\boldsymbol{v}(\mathcal{U}))=\left(\begin{array}{ccccccc}
1 & \bullet & 0 & 0 & \bullet & \bullet & \bullet \\
0 & 0 & 1 & 0 & \bullet & \bullet & \bullet \\
0 & 0 & 0 & 1 & \bullet & \bullet & \bullet
\end{array}\right)
$$

To present the multilevel construction, the following two lemmas are crucial.

Lemma 3.7 [6, Lemma 2] Let $\mathcal{U}, \mathcal{V} \in \mathcal{G}_{q}(n, k), \mathcal{U}=\operatorname{rowspace}(\boldsymbol{U})$ and $\mathcal{V}=\operatorname{rowspace}(\boldsymbol{V})$, where $\boldsymbol{U}, \boldsymbol{V} \in \mathbb{F}_{q}^{k \times n}$ are in reduced row echelon forms. Then

$$
d_{S}(\mathcal{U}, \mathcal{V}) \geq d_{H}(\boldsymbol{v}(\boldsymbol{U}), \boldsymbol{v}(\boldsymbol{V}))
$$


Lemma 3.8 ( 6], [28, Proposition 1.2]) Let $\mathcal{U}, \mathcal{V} \in \mathcal{G}_{q}(n, k), \mathcal{U}=\operatorname{rowspace}(\boldsymbol{U})$ and $\mathcal{V}=$ rowspace $(\boldsymbol{V})$, where $\boldsymbol{U}, \boldsymbol{V} \in \mathbb{F}_{q}^{k \times n}$ are in reduced row echelon forms. If $\boldsymbol{v}(\boldsymbol{U})=\boldsymbol{v}(\boldsymbol{V})$, then

$$
d_{S}(\mathcal{U}, \mathcal{V})=2 d_{R}\left(\boldsymbol{C}_{\boldsymbol{U}}, \boldsymbol{C}_{\boldsymbol{V}}\right)
$$

where $\boldsymbol{C}_{\boldsymbol{U}}$ and $\boldsymbol{C}_{\boldsymbol{V}}$ denote the submatrices of $\boldsymbol{U}$ and $\boldsymbol{V}$, respectively, without the columns of their pivots.

Construction 3.9 (Multilevel construction [6]) Let $\mathcal{C}$ be a binary Hamming code of length $n$, weight $k$ and minimum Hamming distance $2 \delta$. For each codeword $\boldsymbol{C} \in \mathcal{C}$, its echelon Ferrers form is $E F(\boldsymbol{C})$. All dots in $E F(\boldsymbol{C})$ produce a Ferrers diagram $\mathcal{F}_{\boldsymbol{C}}$. If there exists an $\left[\mathcal{F}_{\boldsymbol{C}}, k_{\boldsymbol{C}}, \delta\right]_{q}$ code $\mathcal{D}_{\boldsymbol{C}}$ for each $\boldsymbol{C} \in \mathcal{C}$, then by Lemmas 3.7 and 3.8 , the row spaces of the matrices in $\bigcup_{\boldsymbol{C} \in \mathcal{C}} \mathcal{D}_{\boldsymbol{C}}$ form a $(n, 2 \delta, k)_{q}-C D C$.

\subsection{Combination of parallel construction and multilevel construction}

Although the multilevel construction is effective to construct CDCs, it is not known so far how to pick up identifying vectors such that the resulting CDCs are optimal. Also, only a few infinite families on optimal FDRMCs are known in the literature. The following constructions help to reduce the choice of identifying vectors.

\subsubsection{The first construction}

Construction 3.10 Let $n \geq 2 k$. Suppose that there exists an $\left(n, M_{1}, 2 \delta, k\right)_{q}-C D C$ which is constructed via the multilevel construction satisfying that for any of its identifying vectors $\boldsymbol{v}=(\underbrace{\boldsymbol{v}^{(1)}}_{n-k} \mid \underbrace{\boldsymbol{v}^{(2)}}_{k})$, it holds that wt $\left(\boldsymbol{v}^{(1)}\right) \geq s \geq \delta$, where wt $\left(\boldsymbol{v}^{(1)}\right)$ is the weight of $\boldsymbol{v}^{(1)}$. If there exists a $\left(k \times(n-k), M_{2}, \delta,[0, s-\delta]\right)_{q}-G R M C$, then there exists an $\left(n, M_{1}+M_{2}, 2 \delta, k\right)_{q}-C D C$.

Proof Let $\mathcal{C}_{1}$ be the given $\left(n, M_{1}, 2 \delta, k\right)_{q}$-CDC and $\mathcal{D}$ be a $\left(k \times(n-k), M_{2}, \delta,[0, s-\delta]\right)_{q^{-}}$ GRMC. Set $\mathcal{C}_{2}=\left\{\operatorname{rowspace}\left(\boldsymbol{B} \mid \boldsymbol{I}_{k}\right): \boldsymbol{B} \in \mathcal{D}\right\}$. Let $\mathcal{C}=\mathcal{C}_{1} \cup \mathcal{C}_{2}$. Then $\mathcal{C}$ is an $\left(n, M_{1}+M_{2}, 2 \delta, k\right)_{q}$-CDC.

It suffices to examine the subspace distance of $\mathcal{C}$. For any $\mathcal{U} \in \mathcal{C}_{1}$, let $(\underbrace{\boldsymbol{A}}_{n-k} \mid \underbrace{\boldsymbol{D}}_{k})$ be

the reduced row echelon form of $\mathcal{U}$. We have $\operatorname{rank}(\boldsymbol{A}) \geq s$ by the fact that any identifying vector $\boldsymbol{v}$ satisfies $\operatorname{wt}\left(\boldsymbol{v}^{(1)}\right) \geq s$. For any $\mathcal{V} \in \mathcal{C}_{2}$,

$$
\begin{aligned}
d_{S}(\mathcal{U}, \mathcal{V}) & =2 \cdot \operatorname{rank}\left(\begin{array}{cc}
\boldsymbol{A} & \boldsymbol{D} \\
\boldsymbol{B} & \boldsymbol{I}_{k}
\end{array}\right)-2 k \\
& =2 \cdot \operatorname{rank}\left(\begin{array}{cc}
\boldsymbol{A}-\boldsymbol{D} \boldsymbol{B} & \boldsymbol{O} \\
\boldsymbol{B} & \boldsymbol{I}_{k}
\end{array}\right)-2 k \\
& =2 \cdot \operatorname{rank}(\boldsymbol{A}-\boldsymbol{D} \boldsymbol{B}) \\
& \geq 2 s-2 \cdot \operatorname{rank}(\boldsymbol{D} \boldsymbol{B}) \\
& \geq 2 s-2 \cdot \operatorname{rank}(\boldsymbol{B}) \geq 2 s-2(s-\delta)=2 \delta .
\end{aligned}
$$

For any $\mathcal{U}, \mathcal{V} \in \mathcal{C}_{2}$ and $\mathcal{U} \neq \mathcal{V}$, we know $d_{S}(\mathcal{U}, \mathcal{V}) \geq 2 \delta$ by Lemma 3.8 . 
Construction 3.10 shows a criteria to combine the parallel construction and the multilevel construction to produce CDCs with large size. When $n \geq 2 k+\delta$ and $k \geq 2 \delta$, applying Construction 3.10, in what follows we shall present better lower bounds on $\bar{A}_{q}(n, 2 \delta, k)$ than that in Theorem 2.6.

Lemma 3.11 Let $n \geq 2 k+\delta$ and $k \geq 2 \delta$. Let $q$ be any prime power and

$$
M_{1}=q^{(n-k)(k-\delta+1)} \frac{1-q^{-\left\lfloor\frac{k}{\delta}\right\rfloor \delta^{2}}}{1-q^{-\delta^{2}}}+q^{(n-k-\delta)(k-\delta+1)} .
$$

Then there exists an $\left(n, M_{1}, 2 \delta, k\right)_{q}-C D C$ constructed via the multilevel construction satisfying that for any of its identifying vectors $\boldsymbol{v}=(\underbrace{\boldsymbol{v}^{(1)}}_{n-k} \mid \underbrace{\boldsymbol{v}^{(2)}}_{k})$, it holds that $w t\left(\boldsymbol{v}^{(1)}\right) \geq k$, and this CDC contains a lifted MRD code $\left(n, q^{(n-k)(k-\delta+1)}, 2 \delta, k\right)_{q}-C D C$ as a subset.

Proof We construct the set of identifying vectors of length $n$ as follows:

$$
\mathcal{A}=\{(\underbrace{1 \ldots 1}_{k} \underbrace{0 \ldots 0}_{n-k})\} \cup\{(\boldsymbol{u} \mid \underbrace{1 \ldots 1}_{\delta} \underbrace{0 \ldots 0}_{n-k-\delta}): \boldsymbol{u} \in \mathcal{B}\},
$$

where

$$
\mathcal{B}=\left\{(\underbrace{1 \ldots 1}_{k-\delta} \underbrace{0 \ldots 0}_{\delta}),(\underbrace{1 \ldots 1}_{k-2 \delta} \underbrace{0 \ldots 0}_{\delta} \underbrace{1 \ldots 1}_{\delta}), \ldots,(\underbrace{1 \ldots 1}_{k-\left\lfloor\frac{k}{\delta}\right\rfloor \delta} \underbrace{0 \ldots 0}_{\delta} \underbrace{1 \ldots 1}_{\left(\left\lfloor\frac{k}{\delta}\right\rfloor-1\right) \delta})\right\} .
$$

The size of $\mathcal{B}$ is $\left\lfloor\frac{k}{\delta}\right\rfloor$, and each vector in $\mathcal{B}$ has size $k$ and weight $k-\delta$ (note that the $\delta$ zeros are always shifted to the left by $\delta$ positions). It is readily checked that for any $\boldsymbol{v}, \boldsymbol{v}^{\prime} \in \mathcal{A}, \boldsymbol{v} \neq \boldsymbol{v}^{\prime}$, we have $d_{H}\left(\boldsymbol{v}, \boldsymbol{v}^{\prime}\right) \geq 2 \delta$.

For any identifying vector $\boldsymbol{v}_{i}=(\underbrace{\boldsymbol{v}_{i}^{(1)}}_{n-k} \mid \underbrace{\boldsymbol{v}_{i}^{(2)}}_{k})=(\underbrace{1 \ldots 1}_{k-i \delta} \underbrace{0 \ldots 0}_{\delta} \underbrace{1 \ldots 1}_{i \delta} \underbrace{0 \ldots 0}_{n-k-\delta}) \in \mathcal{A}, 0 \leq$ $i \leq\left\lfloor\frac{k}{\delta}\right\rfloor$, we have $\operatorname{wt}\left(\boldsymbol{v}_{i}^{(1)}\right) \geq k$ because of $n-k \geq k+\delta$. The echelon Ferrers form of $\boldsymbol{v}_{i}$ is

$$
E F\left(\boldsymbol{v}_{i}\right)=\left(\begin{array}{cccc}
\boldsymbol{I}_{k-i \delta} & \mathcal{F}_{1} & \boldsymbol{O}_{1} & \mathcal{F}_{2} \\
\boldsymbol{O}_{2} & \boldsymbol{O}_{3} & \boldsymbol{I}_{i \delta} & \mathcal{F}_{3}
\end{array}\right)
$$

where $\mathcal{F}_{1}$ is a $(k-i \delta) \times \delta$ full Ferrers diagram, $\mathcal{F}_{2}$ is a $(k-i \delta) \times(n-k-\delta)$ full Ferrers diagram, $\mathcal{F}_{3}$ is an $i \delta \times(n-k-\delta)$ full Ferrers diagram, $\boldsymbol{O}_{1}$ is a $(k-i \delta) \times i \delta$ zero matrix, $\boldsymbol{O}_{2}$ is an $i \delta \times(k-i \delta)$ zero matrix and $\boldsymbol{O}_{3}$ is an $i \delta \times \delta$ zero matrix. For $0 \leq i \leq\left\lfloor\frac{k}{\delta}\right\rfloor$, let

$$
\mathcal{H}_{i}=[\underbrace{k-i \delta, \ldots, k-i \delta}_{\delta}, \underbrace{k, \ldots, k}_{n-k-\delta}]
$$

be a $k \times(n-k)$ Ferrers diagram, and

$$
\mathcal{C}_{i}=\left\{\operatorname{rowspace}\left(\begin{array}{cccc}
\boldsymbol{I}_{k-i \delta} & \boldsymbol{D}_{1} & \boldsymbol{O}_{1} & \boldsymbol{D}_{2} \\
\boldsymbol{O}_{2} & \boldsymbol{O}_{3} & \boldsymbol{I}_{i \delta} & \boldsymbol{D}_{3}
\end{array}\right):\left(\begin{array}{cc}
\boldsymbol{D}_{1} & \boldsymbol{D}_{2} \\
\boldsymbol{O} & \boldsymbol{D}_{3}
\end{array}\right) \in \mathcal{C}_{\mathcal{H}_{i}}\right\}
$$

where $\mathcal{C}_{\mathcal{H}_{i}}$ is an $\left[\mathcal{H}_{i},(n-k)(k-\delta+1)-i \delta^{2}, \delta\right]_{q}$ code when $0 \leq i \leq\left\lfloor\frac{k}{\delta}\right\rfloor-1$, and $\mathcal{C}_{\mathcal{H}_{i}}$ is an $\left[\mathcal{H}_{i},(n-k-\delta)(k-\delta+1), \delta\right]_{q}$ code when $i=\left\lfloor\frac{k}{\delta}\right\rfloor$.

Here we need to examine the existence of $\mathcal{C}_{\mathcal{H}_{i}}$. Consider the transpose of $\mathcal{H}_{i}$ : 


$$
\mathcal{H}_{i}^{t}=[\underbrace{n-k-\delta, \ldots, n-k-\delta}_{i \delta}, \underbrace{n-k, \ldots, n-k}_{k-i \delta}] .
$$

For the $(n-k) \times k$ Ferrers diagram $\mathcal{H}_{i}^{t}$, every column of $\mathcal{H}_{i}^{t}$ contains at least $n-k-\delta$ dots. Since $n-k-\delta \geq k$, by Theorem [3.3, there exists an optimal $\left[\mathcal{H}_{i}^{t}, \delta\right]_{q}$ code. Now it remains to analyse its dimension. If $k-i \delta \geq \delta$, i.e, $i \leq\left\lfloor\frac{k}{\delta}\right\rfloor-1$, then by Theorem 3.3 , the optimal dimension is equal to the number of dots in $\mathcal{H}_{i}^{t}$ which are not contained in the rightmost $\delta-1$ columns: $i \delta(n-k-\delta)+(k-i \delta-\delta+1)(n-k)=(n-k)(k-\delta+1)-i \delta^{2}$. Thus there exists an optimal $\left[\mathcal{H}_{i}^{t},(n-k)(k-\delta+1)-i \delta^{2}, \delta\right]_{q}$ code. If $0 \leq k-i \delta<\delta$, i.e, $i=\left\lfloor\frac{k}{\delta}\right\rfloor$, then the optimal dimension is $(n-k-\delta)(k-\delta+1)$. Thus there exists an optimal $\left[\mathcal{H}_{i}^{t},(n-k-\delta)(k-\delta+1), \delta\right]_{q}$ code.

Let $\mathcal{C}=\bigcup_{i=0}^{\left\lfloor\frac{k}{\delta}\right\rfloor} \mathcal{C}_{i}$. Then

$$
\begin{aligned}
|\mathcal{C}| & =\sum_{i=0}^{\left\lfloor\frac{k}{\delta}\right\rfloor-1} q^{(n-k)(k-\delta+1)-i \delta^{2}}+q^{(n-k-\delta)(k-\delta+1)} \\
& =q^{(n-k)(k-\delta+1)} \frac{1-q^{-\left\lfloor\frac{k}{\delta}\right\rfloor \delta^{2}}}{1-q^{-\delta^{2}}}+q^{(n-k-\delta)(k-\delta+1)} .
\end{aligned}
$$

For any $\mathcal{U} \in \mathcal{C}_{i}$ and $\mathcal{V} \in \mathcal{C}_{j}, 0 \leq i, j \leq\left\lfloor\frac{k}{\delta}\right\rfloor, i \neq j$, by Lemma 3.7, we have $d_{s}(\mathcal{U}, \mathcal{V}) \geq$ $d_{H}\left(\boldsymbol{v}_{i}, \boldsymbol{v}_{j}\right) \geq 2 \delta$. For any $\mathcal{U}, \mathcal{V} \in \mathcal{C}_{i}, 0 \leq i \leq\left\lfloor\frac{k}{\delta}\right\rfloor$, by Lemma 3.8, we have $d_{s}(\mathcal{U}, \mathcal{V}) \geq 2 \delta$. Thus $\mathcal{C}$ is an $\left(n, M_{1}, 2 \delta, k\right)_{q}$-CDC. This CDC contains a lifted MRD code $\left(n, q^{(n-k)(k-\delta+1)}\right.$, $2 \delta, k)_{q}$-CDC as a subset which comes from the identifying vector $(\underbrace{1 \ldots 1}_{k} \underbrace{0 \ldots 0}_{n-k})$.

Applying Lemma 3.11 and Construction 3.10 with $s=k$, we obtain the following result.

Theorem 3.12 Let $n \geq 2 k+\delta$ and $k \geq 2 \delta$. Then

$$
\begin{aligned}
\bar{A}_{q}(n, 2 \delta, k) \geq q^{(n-k)(k-\delta+1)} \frac{1-q^{-\left\lfloor\frac{k}{\delta}\right\rfloor \delta^{2}}}{1-q^{-\delta^{2}}} & +q^{(n-k-\delta)(k-\delta+1)} \\
& +A_{q}^{R}(k \times(n-k), \delta,[0, k-\delta]) .
\end{aligned}
$$

Theorem 3.12 together with the use of Proposition 2.4 provides many new constant dimension codes with larger size than the previously best known codes in [16]. We list some of them in Table 1 in Appendix B.

When $\delta=2$, Theorem 3.12 can be improved by using the following CDCs constructed in [24].

Lemma 3.13 [24, Construction B, Corollary 27] Let $n \geq 2 k+2$ and $k \geq 4$. Let $q$ be any prime power and

$$
M_{1}=\sum_{j=1}^{\left\lfloor\frac{n-2}{k}\right\rfloor-1}\left(q^{(k-1)(n-j k)}+\frac{\left(q^{2(k-2)}-1\right)\left(q^{2(n-j k-1)}-1\right)}{\left(q^{4}-1\right)^{2}} q^{(k-3)(n-j k-2)+4}\right) .
$$

Then there exists an $\left(n, M_{1}, 4, k\right)_{q}$-CDC constructed via the multilevel construction satisfying that for any of its identifying vectors $\boldsymbol{v}=(\underbrace{\boldsymbol{v}^{(1)}}_{n-k} \mid \underbrace{\boldsymbol{v}^{(2)}}_{k})$, it holds that wt $\left(\boldsymbol{v}^{(1)}\right) \geq k-2$, and this CDC contains a lifted MRD code $\left(n, q^{(n-k)(k-\delta+1)}, 4, k\right)_{q}-C D C$ as a subset. 
Proof (sketch only) We here employ the same set of identifying vectors as those in Construction B of [24] (note that the identifying vectors are exhibited in [24] recursively, and here we list all of them explicitly):

$$
\begin{aligned}
& \mathcal{A}=\left\{(\underbrace{0 \ldots 0}_{i k} \underbrace{1 \ldots 1}_{k} \underbrace{0 \ldots 0}_{n-(i+1) k}): 0 \leq i \leq\left\lfloor\frac{n-2}{k}\right\rfloor-2\right\} \cup \\
& \left\{(\underbrace{0 \ldots 0}_{i k}|\underbrace{\boldsymbol{w}}_{k}| \underbrace{\boldsymbol{z}}_{n-(i+1) k}): \boldsymbol{w} \in \mathcal{B}, \boldsymbol{z} \in \mathcal{D}_{i+1}, 0 \leq i \leq\left\lfloor\frac{n-2}{k}\right\rfloor-2\right\},
\end{aligned}
$$

where

$$
\mathcal{B}=\{(\underbrace{1 \ldots 1}_{k-2} 00),(\underbrace{1 \ldots 1}_{k-4} 0011), \ldots, \boldsymbol{u}\}, \quad \boldsymbol{u}= \begin{cases}(00 \underbrace{11 \ldots 1}_{k-2}), & \text { if } k \text { is even; } \\ (100 \underbrace{1 \ldots 1}_{k-3}), & \text { if } k \text { is odd }\end{cases}
$$

and

$$
\begin{gathered}
\mathcal{D}_{i+1}=\left\{(11 \underbrace{00 \ldots 00}_{n-(i+1) k-2}),(0011 \underbrace{00 \ldots 00}_{n-(i+1) k-4}), \ldots, \boldsymbol{u}^{\prime}\right\}, \\
\boldsymbol{u}^{\prime}=\left\{\begin{array}{l}
(\underbrace{00 \ldots 00}_{n-(i+1) k-2} 11), \text { if } n-(i+1) k \text { is even } ; \\
(\underbrace{00 \ldots 00}_{n-(i+1) k-3} 110), \text { if } n-(i+1) k \text { is odd } .
\end{array}\right.
\end{gathered}
$$

The size of $\mathcal{B}$ is $\left\lfloor\frac{k}{2}\right\rfloor$, and each vector in $\mathcal{B}$ has size $k$ and weight $k-2$ (note that the two zeros are always shifted to the left by two positions). The size of $\mathcal{D}_{i+1}$ is $\left\lfloor\frac{n-(i+1) k}{2}\right\rfloor$, and each vector in $\mathcal{D}_{i+1}$ has size $n-(i+1) k$ and weight 2 (note that the two ones are always shifted to the left by two positions).

Since $i \leq\left\lfloor\frac{n-2}{k}\right\rfloor-2$, we have $n-k \geq(i+1) k$. Then for any identifying vector $\boldsymbol{v}=(\underbrace{\boldsymbol{v}^{(1)}}_{n-k} \mid \underbrace{\boldsymbol{v}^{(2)}}_{k})$ shown above, one can check that $\operatorname{wt}\left(\boldsymbol{v}^{(1)}\right) \geq k-2$. By Construction B and Corollary 27 in [24, these identifying vectors generate an $\left(n, M_{1}, 4, k\right)_{q}$-CDC. This CDC contains a lifted MRD code $\left(n, q^{(n-k)(k-\delta+1)}, 4, k\right)_{q}$-CDC as a subset which comes from the identifying vector $(\underbrace{1 \ldots 1}_{k} \underbrace{0 \ldots 0}_{n-k})$.

Theorem 3.14 Let $n \geq 2 k+2$ and $k \geq 4$. Then

$$
\begin{aligned}
\bar{A}_{q}(n, 4, k) \geq & \sum_{j=1}^{\left\lfloor\frac{n-2}{k}\right\rfloor-1}\left(q^{(k-1)(n-j k)}+\frac{\left(q^{2(k-2)}-1\right)\left(q^{2(n-j k-1)}-1\right)}{\left(q^{4}-1\right)^{2}} q^{(k-3)(n-j k-2)+4}\right) \\
& +A_{q}^{R}(k \times(n-k), 2,[0, k-4]) .
\end{aligned}
$$

Proof Applying Lemma 3.13 and Construction 3.10 with $s=k-2$. 
With the aid of a computer, we compared the values of $\bar{A}_{q}(n, 4, k)$ from Theorems 3.12 and 3.14. It seems that when $k>4$ and $n$ is large enough, Theorem 3.14 always produces better lower bound on $\bar{A}_{q}(n, 4, k)$ than that in Theorem 3.12 (see Table 2 in Appendix B for example).

More specially, for $\delta=4$ and $k=5$, Silberstein and Trautmann in 24] presented an $\left(n, M_{1}, 4,5\right)_{q}$-CDC with larger size than that in Lemma 3.13 by choosing identifying vectors more carefully.

Lemma 3.15 [24, Construction C-5, Theorem 35] Let $n \geq 12$ and $q$ be any prime power. If $x$ is even, then let

$$
\begin{aligned}
M(x) & :=q^{4(x-5)}+\left(q^{2 x-10}+q^{2 x-14}\right)\left(q^{2 x-14}+\frac{x-8}{2} q^{x-9}\right)+\left(q^{2 x-11}+q^{2 x-13}\right) \\
& \times\left(\frac{x-8}{2} q^{x-10}+q^{2 x-15}\right)+\left(q^{2 x-12}+q^{2 x-13}\right)\left(2 q^{2 x-16}+\frac{x-10}{2} q^{x-11}\right) \\
& +\left(q^{2 x-12}+q^{2 x-14}\right) \times\left[\sum_{i=3}^{\min \left\{\left\lceil\frac{q}{2}\right\rceil+2,\left\lfloor\frac{x-5}{2}\right\rfloor\right\}}\left(i q^{2 x-2 i-12}+\left(\frac{x-6}{2}-i\right) q^{x-2 i-7}\right)\right. \\
& \left.+\sum_{i=2}^{\min \left\{\left\lfloor\frac{q}{2}\right\rfloor+2,\left\lceil\frac{x-7}{2}\right\rceil\right\}}\left(i q^{2 x-2 i-13}+\left(\frac{x-6}{2}-i\right) q^{x-2 i-8}\right)\right] .
\end{aligned}
$$

If $x$ is odd, then let

$$
\begin{aligned}
M(x) & :=q^{4(x-5)}+\left(q^{2 x-10}+q^{2 x-14}\right)\left(q^{2 x-14}+\frac{x-9}{2} q^{x-8}+q^{\frac{x-9}{2}}\right)+\left(q^{2 x-11}+q^{2 x-13}\right) \\
& \times\left(\frac{x-9}{2} q^{x-9}+q^{2 x-15}+q^{x-8}\right)+\left(q^{2 x-12}+q^{2 x-13}\right)\left(q^{2 x-16}+\frac{x-11}{2} q^{x-10}+q^{\frac{x-11}{2}}\right) \\
& +\left(q^{2 x-12}+q^{2 x-14}\right) \times\left[\sum_{i=3}^{\min \left\{\left\lceil\frac{q}{2}\right\rceil+2,\left\lfloor\frac{x-5}{2}\right\rfloor\right\}}\left(i q^{2 x-2 i-12}+\left(\frac{x-7}{2}-i\right) q^{x-2 i-6}+q^{\frac{x-7}{2}-i}\right)\right. \\
& \left.+\sum_{i=2} \sum^{\min \left\{\left\lfloor\frac{q}{2}\right\rfloor+2,\left\lceil\frac{x-7}{2}\right\rceil\right\}}\left(i q^{2 x-2 i-13}+\left(\frac{x-7}{2}-i\right) q^{x-2 i-7}+q^{x-i-7}\right)\right] .
\end{aligned}
$$

Let

$$
M_{1}=\sum_{j=0}^{\left\lfloor\frac{n-12}{5}\right\rfloor} M(n-5 j) .
$$

Then there exists an $\left(n, M_{1}, 4,5\right)_{q}-C D C$ constructed via the multilevel construction satisfying that for any of its identifying vectors $\boldsymbol{v}=(\underbrace{\boldsymbol{v}^{(1)}}_{n-5} \mid \underbrace{\boldsymbol{v}^{(2)}}_{5})$, it holds that $w t\left(\boldsymbol{v}^{(1)}\right) \geq 3$, and this CDC contains a lifted MRD code $\left(n, q^{4(n-5)}, 4,5\right)_{q^{-}} C D C$ as a subset.

Proof (sketch only) We here employ the same set of identifying vectors as those in Construction C-5 of [24] (note that the identifying vectors are exhibited in [24] recursively, 
and here we list all of them explicitly):

$$
\begin{aligned}
& \{(11111 \mid \underbrace{0 \ldots 0)}_{n-5}\} \cup \\
& \left\{(\underbrace{0 \ldots 0}_{5 j}|11100| \boldsymbol{u}),(\underbrace{0 \ldots 0}_{5 j}|10011| \boldsymbol{u}): \boldsymbol{u} \in P_{j,\left\lceil\frac{n-5 j-5}{2}\right\rceil+1}, 0 \leq j \leq\left\lfloor\frac{n-12}{5}\right\rfloor\right\} \cup \\
& \left\{(\underbrace{0 \ldots 0}_{5 j}|11010| \boldsymbol{u}),(\underbrace{0 \ldots 0}_{5 j}|01101| \boldsymbol{u}): \boldsymbol{u} \in P_{j, 2}, 0 \leq j \leq\left\lfloor\frac{n-12}{5}\right\rfloor\right\} \cup \\
& \left\{(\underbrace{0 \ldots 0}_{5 j}|01110| \boldsymbol{u}),(\underbrace{0 \ldots 0}_{5 j}|10101| \boldsymbol{u}): \boldsymbol{u} \in P_{j,\left\lceil\frac{n-5 j-5}{2}\right\rceil+2}, 0 \leq j \leq\left\lfloor\frac{n-12}{5}\right\rfloor\right\} \cup \\
& \left\{(\underbrace{0 \ldots 0}_{5 j}|00111| \boldsymbol{u}),(\underbrace{0 \ldots 0}_{5 j}|11001| \boldsymbol{u}): \boldsymbol{u} \in P_{j, 3}, 0 \leq j \leq\left\lfloor\frac{n-12}{5}\right\rfloor\right\} \cup \\
& \left\{(\underbrace{0 \ldots 0}_{5 j}|10110| \boldsymbol{u}),(\underbrace{0 \ldots 0}_{5 j}|01011| \boldsymbol{u}): 0 \leq j \leq\left\lfloor\frac{n-12}{5}\right\rfloor,\right. \\
& \boldsymbol{u} \in\left(\bigcup_{i=3}^{\min \left\{\left\lceil\frac{q}{2}\right\rceil+2,\left\lfloor\frac{n-5 j-5}{2}\right\rfloor\right\}} P_{j,\left\lceil\frac{n-5 j-5}{2}\right\rceil+i} \cup\left(\bigcup_{i=4}^{\min \left\{\left\lfloor\frac{q}{2}\right\rfloor+3,\left\lceil\frac{n-5 j-5}{2}\right\rceil\right\}} P_{j, i}\right)\right\},
\end{aligned}
$$

where each vector in $P_{j, l}$ has size $n-5 j-5$ and weight 2 ; if $n-5 j-5$ is even, then the positions of ones in vectors from $P_{j, 2}, \ldots, P_{j, n-5 j-5}$ correspond to a one-factorization of the complete graph $K_{n-5 j-5}$; if $n-5 j-5$ is odd, then the positions of ones in vectors from $P_{j, 1}, P_{j, 2}, \ldots, P_{j, n-5 j-5}$ correspond to a near one-factorization of the complete graph $K_{n-5 j-5}$ (see [24, Construction C-5] for more details).

Since $j \leq\left\lfloor\frac{n-12}{5}\right\rfloor$, we have $5 j+5<n-5$. Then for any identifying vector $\boldsymbol{v}=$ $(\underbrace{\boldsymbol{v}^{(1)}}_{n-5} \mid \underbrace{\boldsymbol{v}^{(2)}}_{5})$ shown above, one can check that $\operatorname{wt}\left(\boldsymbol{v}^{(1)}\right) \geq 3$. By Construction C-5 and Theorem 35 in 24, these identifying vectors generate an $\left(n, M_{1}, 4,5\right)_{q}$-CDC. This CDC contains a lifted MRD code $\left(n, q^{4(n-5)}, 4,5\right)_{q}$-CDC as a subset which comes from the identifying vector $(11111 \mid \underbrace{0 \ldots 0}_{n-5})$.

Theorem 3.16 Let $M_{1}$ be as in Lemma 3.15 and $n \geq 12$. Then

$$
\bar{A}_{q}(n, 4,5) \geq M_{1}+q^{4}+q^{3}+q^{2}+q+1 .
$$

Proof Applying Lemma 3.15 and Construction 3.10 with $s=3$, we have

$$
\bar{A}_{q}(n, 4,5) \geq M_{1}+A_{q}^{R}(5 \times(n-5), 2,\{0,1\}) .
$$

By Proposition 2.4 and Theorem 2.2, one can check that

$$
A_{q}^{R}(5 \times(n-5), 2,\{0,1\}) \geq q^{4}+q^{3}+q^{2}+q+1 .
$$

This completes the proof.

Theorem 3.16 provides many new $(n, 4,5)_{q}$-CDCs with larger size than the previously best known codes in [16. We list some of them in Table 2 in Appendix B. Compared with Theorem 3.16. Theorems 3.12 and 3.14 produce worse lower bounds of $\bar{A}_{q}(n, 4,5)$ for those $n$ in Table 2 . 


\subsubsection{The second construction}

In Construction 3.10, we start from a multilevel construction, and then choose an appropriate parallel construction based on the identifying vectors in the multilevel construction. Actually, we can also start from a parallel construction, and then choose appropriate identifying vectors to use the multilevel construction.

Construction 3.17 Let $n \geq 2 k$ and $k \geq 2 \delta$. If there exists $a(k \times(n-k), M, \delta,[0, k-\delta])_{q}$ $G R M C$, then there exists an $\left(n, q^{(n-k)(k-\delta+1)}+M+q^{\max \left\{l_{1}, l_{2}\right\}}+q^{(n-k-\delta)(k-2 \delta+1)}, 2 \delta, k\right)_{q^{-}}$ $C D C$, where

$$
l_{1}=\left\{\begin{array}{l}
(k-\delta) \delta+n-k-\delta, \quad \text { if } n \geq k+3 \delta \\
\delta(n-4 \delta+2), \quad \text { if } n<k+3 \delta
\end{array}\right.
$$

and

$$
l_{2}=\max _{1 \leq j \leq \delta-1}\{\min \{(\delta-j+1)(k-\delta),(j+1)(n-k-\delta)\}\} .
$$

This CDC contains a lifted MRD code $\left(n, q^{(n-k)(k-\delta+1)}, 2 \delta, k\right)_{q}-C D C$ as a subset.

Proof Let $\mathcal{D}_{1}$ be an $\operatorname{MRD}[k \times(n-k), \delta]_{q}$ code and $\mathcal{D}_{2}$ be a $(k \times(n-k), M, \delta,[0, k-\delta])_{q^{-}}$ GRMC. Set

$$
\mathcal{C}_{1}=\left\{\operatorname{rowspace}\left(\boldsymbol{I}_{k} \mid \boldsymbol{A}\right): \boldsymbol{A} \in \mathcal{D}_{1}\right\}
$$

and

$$
\mathcal{C}_{2}=\left\{\operatorname{rowspace}\left(\boldsymbol{B} \mid \boldsymbol{I}_{k}\right): \boldsymbol{B} \in \mathcal{D}_{2}\right\} .
$$

Since $n \geq 2 k,\left|\mathcal{C}_{1}\right|=q^{(n-k)(k-\delta+1)}$. Note that the identifying vector of each codeword in $\mathcal{C}_{1}$ is $\boldsymbol{n}=(\underbrace{1 \ldots 1}_{k} \underbrace{0 \ldots 0}_{n-k})$. Now we take two new identifying vectors $\boldsymbol{n}_{1}$ and $\boldsymbol{n}_{2}$.

Step 1. Take

$$
\boldsymbol{n}_{1}=(\underbrace{1 \ldots 1}_{k-\delta} \underbrace{0 \ldots 0}_{\delta} \underbrace{1 \ldots 1}_{\delta} \underbrace{0 \ldots 0}_{n-k-\delta}) .
$$

Note that $d_{H}\left(\boldsymbol{n}, \boldsymbol{n}_{1}\right)=2 \delta$. Then

$$
E F\left(\boldsymbol{n}_{1}\right)=\left(\begin{array}{cccc}
\boldsymbol{I}_{k-\delta} & \mathcal{F}_{1} & \boldsymbol{O}_{1} & \mathcal{F}_{3} \\
\boldsymbol{O}_{2} & \boldsymbol{O}_{3} & \boldsymbol{I}_{\delta} & \mathcal{F}_{2}
\end{array}\right)
$$

where $\mathcal{F}_{1}$ is a $(k-\delta) \times \delta$ full Ferrers diagram, $\mathcal{F}_{2}$ is a $\delta \times(n-k-\delta)$ full Ferrers diagram, $\mathcal{F}_{3}$ is a $(k-\delta) \times(n-k-\delta)$ full Ferrers diagram, $\boldsymbol{O}_{1}$ is a $(k-\delta) \times \delta$ zero matrix, $\boldsymbol{O}_{2}$ is a $\delta \times(k-\delta)$ zero matrix and $\boldsymbol{O}_{3}$ is a $\delta \times \delta$ zero matrix. Let

$$
\mathcal{F}=\left(\begin{array}{cc}
\mathcal{F}_{1} & \mathcal{F}_{3} \\
& \mathcal{F}_{2}
\end{array}\right)
$$

be a $k \times(n-k)$ Ferrers diagram and

$$
\mathcal{C}_{3}=\left\{\operatorname{rowspace}\left(\begin{array}{cccc}
\boldsymbol{I}_{k-\delta} & \boldsymbol{D}_{1} & \boldsymbol{O}_{1} & \boldsymbol{D}_{3} \\
\boldsymbol{O}_{2} & \boldsymbol{O}_{3} & \boldsymbol{I}_{\delta} & \boldsymbol{D}_{2}
\end{array}\right):\left(\begin{array}{cc}
\boldsymbol{D}_{1} & \boldsymbol{D}_{3} \\
\boldsymbol{O}_{3} & \boldsymbol{D}_{2}
\end{array}\right) \in \mathcal{D}_{\mathcal{F}}\right\}
$$

where $\mathcal{D}_{\mathcal{F}}$ is a Ferrers diagram rank-metric code in $\mathcal{F}$ with minimum rank distance $\delta$, which will be constructed as follows in two different ways.

(1) We claim that there exists an $[\mathcal{F},(k-\delta) \delta+n-k-\delta, \delta]_{q}$ code $\mathcal{D}_{\mathcal{F}}^{\prime}$ when $n \geq k+3 \delta$, and there exists an $[\mathcal{F}, \delta(n-4 \delta+2), \delta]_{q}$ code $\mathcal{D}_{\mathcal{F}}^{\prime}$ when $2 k \leq n<k+3 \delta$.

To examine the existence of such FDRMC codes, take an $(n-k-\delta) \times 2 \delta$ Ferrers diagram 


$$
\mathcal{F}_{12}=\left(\mathcal{F}_{1} \mid \mathcal{F}_{2}^{t}\right)=[\underbrace{k-\delta, \ldots, k-\delta}_{\delta}, \underbrace{n-k-\delta, \ldots, n-k-\delta}_{\delta}]
$$

Note that

$$
\mathcal{F}_{12}^{t}=[\underbrace{\delta, \ldots, \delta}_{n-2 k}, \underbrace{2 \delta, \ldots, 2 \delta}_{k-\delta}]
$$

By Theorem 3.3, when $n-k-\delta \geq 2 \delta$, i.e., $n \geq k+3 \delta$, there exists an optimal $\left[\mathcal{F}_{12},(k-\right.$ $\delta) \delta+n-k-\delta, \delta]_{q}$ code, and when $n<k+3 \delta$, since $k-\delta \geq \delta$, there exists an optimal $\left[\mathcal{F}_{12}^{t}, \delta(n-4 \delta+2), \delta\right]_{q}$ code, which yields an $\left[\mathcal{F}_{12}, \delta(n-4 \delta+2), \delta\right]_{q}$ code. Now applying Theorem 3.5, we obtain an $[\mathcal{F},(k-\delta) \delta+n-k-\delta, \delta]_{q}$ code $\mathcal{D}_{\mathcal{F}}^{\prime}$ when $n \geq k+3 \delta$, and an $[\mathcal{F}, \delta(n-4 \delta+2), \delta]_{q}$ code $\mathcal{D}_{\mathcal{F}}^{\prime}$ when $2 k \leq n<k+3 \delta$. Note that each codeword in $\mathcal{D}_{\mathcal{F}}^{\prime}$ is of the form $\left(\begin{array}{cc}* & \boldsymbol{O}_{4} \\ \boldsymbol{O}_{3} & *\end{array}\right)$, where $\boldsymbol{O}_{4}$ is a $(k-\delta) \times(n-k-\delta)$ zero matrix.

(2) We claim that there exists an

$$
\left[\mathcal{F}, \max _{1 \leq j \leq \delta-1}\{\min \{(\delta-j+1)(k-\delta),(j+1)(n-k-\delta)\}\}, \delta\right]_{q}
$$

code $\mathcal{D}_{\mathcal{F}}^{\prime \prime}$. Its existence comes from Theorem 3.4 by using an optimal $\left[\mathcal{F}_{1},(\delta-j+1)(k-\right.$ $\delta), j]_{q}$ code, i.e., an $\operatorname{MRD}[(k-\delta) \times \delta, j]_{q}$ code, and an optimal $\left[\mathcal{F}_{2},(j+1)(n-k-\delta), \delta-j\right]_{q}$ code, i.e., an $\operatorname{MRD}[\delta \times(n-k-\delta), \delta-j]_{q}$ code (note that $\delta \leq n-k-\delta$ since $n \geq 2 k$ and $k \geq 2 \delta)$. Each codeword in $\mathcal{D}_{\mathcal{F}}^{\prime \prime}$ is of the form $\left(\begin{array}{cc}* & \boldsymbol{O}_{4} \\ \boldsymbol{O}_{3} & *\end{array}\right)$.

Let $l_{1}$ and $l_{2}$ be as in the assumption. If $l_{1} \geq l_{2}$, then take $\mathcal{D}_{\mathcal{F}}=\mathcal{D}_{\mathcal{F}}^{\prime}$. Otherwise, take $\mathcal{D}_{\mathcal{F}}=\mathcal{D}_{\mathcal{F}}^{\prime \prime}$. Thus $\left|\mathcal{C}_{3}\right|=\left|\mathcal{D}_{\mathcal{F}}\right|=q^{\max \left\{l_{1}, l_{2}\right\}}$. Note that $\boldsymbol{D}_{3}=\boldsymbol{O}_{4}$ in both cases.

Step 2. Take

$$
\boldsymbol{n}_{2}=(\underbrace{1 \ldots 1}_{k-2 \delta} \underbrace{0 \ldots 0}_{\delta} \underbrace{1 \ldots 1}_{\delta} \underbrace{1 \ldots 1}_{\delta} \underbrace{0 \ldots 0}_{n-k-\delta}) .
$$

Note that $d_{H}\left(\boldsymbol{n}, \boldsymbol{n}_{2}\right)=d_{H}\left(\boldsymbol{n}_{1}, \boldsymbol{n}_{2}\right)=2 \delta$. Then

$$
E F\left(\boldsymbol{n}_{2}\right)=\left(\begin{array}{ccccc}
\boldsymbol{I}_{k-2 \delta} & \mathcal{F}_{4} & \boldsymbol{O}_{5} & \boldsymbol{O}_{5} & \mathcal{F}_{5} \\
\boldsymbol{O}_{6} & \boldsymbol{O}_{7} & \boldsymbol{I}_{\delta} & \boldsymbol{O}_{7} & \mathcal{F}_{6} \\
\boldsymbol{O}_{6} & \boldsymbol{O}_{7} & \boldsymbol{O}_{7} & \boldsymbol{I}_{\delta} & \mathcal{F}_{7}
\end{array}\right)
$$

where $\mathcal{F}_{4}$ is a $(k-2 \delta) \times \delta$ full Ferrers diagram, $\mathcal{F}_{5}$ is a $(k-2 \delta) \times(n-k-\delta)$ full Ferrers diagram, $\mathcal{F}_{6}$ and $\mathcal{F}_{7}$ are $\delta \times(n-k-\delta)$ full Ferrers diagrams, $\boldsymbol{O}_{5}$ is a $(k-2 \delta) \times \delta$ zero matrix, $\boldsymbol{O}_{6}$ is a $\delta \times(k-2 \delta)$ zero matrix and $\boldsymbol{O}_{7}$ is a $\delta \times \delta$ zero matrix. Let

$$
\mathcal{C}_{4}=\left\{\operatorname{rowspace}\left(\begin{array}{ccccc}
\boldsymbol{I}_{k-2 \delta} & \boldsymbol{O}_{5} & \boldsymbol{O}_{5} & \boldsymbol{O}_{5} & \boldsymbol{D}_{4} \\
\boldsymbol{O}_{6} & \boldsymbol{O}_{7} & \boldsymbol{I}_{\delta} & \boldsymbol{O}_{7} & \boldsymbol{O}_{8} \\
\boldsymbol{O}_{6} & \boldsymbol{O}_{7} & \boldsymbol{O}_{7} & \boldsymbol{I}_{\delta} & \boldsymbol{D}_{5}
\end{array}\right):\left(\begin{array}{c}
\boldsymbol{D}_{4} \\
\boldsymbol{D}_{5}
\end{array}\right) \in \mathcal{D}_{3}\right\},
$$

where $\mathcal{D}_{3}$ is an $\operatorname{MRD}[(k-\delta) \times(n-k-\delta), \delta]_{q}$ code. Since $n-k \geq k,\left|\mathcal{C}_{4}\right|=\left|\mathcal{D}_{3}\right|=$ $q^{(n-k-\delta)(k-2 \delta+1)}$.

Let $\mathcal{C}=\mathcal{C}_{1} \cup \mathcal{C}_{2} \cup \mathcal{C}_{3} \cup \mathcal{C}_{4}$. Then $\mathcal{C}$ is an $\left(n, q^{(n-k)(k-\delta+1)}+M+q^{\max \left\{l_{1}, l_{2}\right\}}+\right.$ $\left.q^{(n-k-\delta)(k-2 \delta+1)}, 2 \delta, k\right)_{q}$-CDC.

It suffices to examine the subspace distance of $\mathcal{C}$. Let $\mathcal{U}_{i} \in \mathcal{C}_{i}$ for $1 \leq i \leq 4$. Since $d_{H}\left(\boldsymbol{n}, \boldsymbol{n}_{1}\right)=d_{H}\left(\boldsymbol{n}, \boldsymbol{n}_{2}\right)=d_{H}\left(\boldsymbol{n}_{1}, \boldsymbol{n}_{2}\right)=2 \delta$, by Lemma 3.7, $d_{S}\left(\mathcal{U}_{1}, \mathcal{U}_{3}\right), d_{S}\left(\mathcal{U}_{1}, \mathcal{U}_{4}\right)$ and 
$d_{S}\left(\mathcal{U}_{3}, \mathcal{U}_{4}\right)$ are all no less than $2 \delta$. Applying Construction 3.10 with $s=k$, we have $d_{S}\left(\mathcal{U}_{1}, \mathcal{U}_{2}\right) \geq 2 \delta$. Since $k \geq 2 \delta$,

$$
\begin{aligned}
d_{S}\left(\mathcal{U}_{2}, \mathcal{U}_{3}\right) & =2 \cdot \operatorname{rank}\left(\begin{array}{ccc:c}
\boldsymbol{I}_{k-\delta} & \boldsymbol{D}_{1} & \boldsymbol{O}_{9} & \boldsymbol{O}_{10} \\
\boldsymbol{O}_{2} & \boldsymbol{O}_{3} & \boldsymbol{D}_{6} & \boldsymbol{D}_{7} \\
\hdashline & \boldsymbol{B} & \boldsymbol{I}_{k}
\end{array}\right)-2 k \\
& \geq 2\left(\operatorname{rank}\left(\begin{array}{ccc}
\boldsymbol{I}_{k-\delta} & \boldsymbol{D}_{1} & \boldsymbol{O}_{9}
\end{array}\right)+\operatorname{rank}\left(\boldsymbol{I}_{k}\right)\right)-2 k=2(k-\delta) \geq 2 \delta,
\end{aligned}
$$

where $(\underbrace{\boldsymbol{O}_{9}}_{n-2 k} \mid \underbrace{\boldsymbol{O}_{10}}_{k})=\left(\boldsymbol{O}_{1} \mid \boldsymbol{O}_{4}\right)$ and $(\underbrace{\boldsymbol{D}_{6}}_{n-2 k} \mid \underbrace{\boldsymbol{D}_{7}}_{k})=\left(\boldsymbol{I}_{\delta} \mid \boldsymbol{D}_{2}\right)$. Similarly we have

$$
d_{S}\left(\mathcal{U}_{2}, \mathcal{U}_{4}\right)=2 \cdot \operatorname{rank}\left(\begin{array}{cccc:c}
\boldsymbol{I}_{k-2 \delta} & \boldsymbol{O}_{5} & \boldsymbol{O}_{5} & \boldsymbol{D}_{8} & \boldsymbol{D}_{9} \\
\boldsymbol{O}_{6} & \boldsymbol{O}_{7} & \boldsymbol{I}_{\delta} & \boldsymbol{O}_{11} & \boldsymbol{O}_{12} \\
\boldsymbol{O}_{6} & \boldsymbol{O}_{7} & \boldsymbol{O}_{7} & \boldsymbol{D}_{10} & \boldsymbol{D}_{11} \\
\hdashline & \boldsymbol{B} & & \boldsymbol{I}_{k}
\end{array}\right)-2 k \geq 2 \delta,
$$

where $(\underbrace{\boldsymbol{D}_{8}}_{n-2 k} \mid \underbrace{\boldsymbol{D}_{9}}_{k})=\left(\boldsymbol{O}_{5} \mid \boldsymbol{D}_{4}\right),(\underbrace{\boldsymbol{O}_{11}}_{n-2 k} \mid \underbrace{\boldsymbol{O}_{12}}_{k})=\left(\boldsymbol{O}_{7} \mid \boldsymbol{O}_{8}\right)$ and $(\underbrace{\boldsymbol{D}_{10}}_{n-2 k} \mid \underbrace{\boldsymbol{D}_{11}}_{k})=\left(\boldsymbol{I}_{\delta} \mid \boldsymbol{D}_{5}\right)$. Finally, by Lemma 3.8, for any $\mathcal{U}, \mathcal{V} \in \mathcal{C}_{i}$ and $\mathcal{U} \neq \mathcal{V}, i \in\{1,2,3,4\}, d_{S}(\mathcal{U}, \mathcal{V}) \geq 2 \delta$.

In the proof of Construction 3.17, three identifying vectors are used: $(\underbrace{1 \ldots 1}_{k} \underbrace{0 \ldots 0}_{n-k})$, $(\underbrace{1 \ldots 1}_{k-\delta} \underbrace{0 \ldots 0}_{\delta} \underbrace{1 \ldots 1}_{\delta} \underbrace{0 \ldots 0}_{n-k-\delta})$ and $(\underbrace{1 \ldots 1}_{k-2 \delta} \underbrace{0 \ldots 0}_{\delta} \underbrace{1 \ldots 1}_{\delta} \underbrace{1 \ldots 1}_{\delta} \underbrace{0 \ldots 0}_{n-k-\delta})$. Since $n \geq 2 k$, for each identifying vectors $\boldsymbol{v}=(\underbrace{\boldsymbol{v}^{(1)}}_{n-k} \mid \underbrace{\boldsymbol{v}^{(2)}}_{k})$, it holds that $w t\left(\boldsymbol{v}^{(1)}\right) \geq k-\delta$. Therefore, if we apply Construction 3.10 via the three identifying vectors to produce CDCs, then we have to construct a $\left(k \times(n-k), M_{2}, \delta,[0, k-2 \delta]\right)_{q}$-GRMC. However, in Construction [3.17, we can use a $(k \times(n-k), M, \delta,[0, k-\delta])_{q}$-GRMC. Generally $M \geq M_{2}$. From this point of view, Construction 3.17 is better than Construction 3.10, But in Construction 3.10 , one can choose other identifying vectors flexibly to change the details of the multilevel construction. From this point of view, Construction 3.10 is better. Anyway, compared with Construction 3.10, Construction 3.17 is easier to be used since its statement does not rely on the choice of identifying vectors.

Construction 3.17 together with the use of Proposition 2.4 provides many new constant dimension codes with larger size than the previously best known codes in [16]. We list some of them in Table 3 in Appendix B. Especially, when $n=2 k=4 \delta$, we obtain new lower bound on $\bar{A}_{q}(n, 2 \delta, k)$.

Corollary $3.18 \bar{A}_{q}(4 \delta, 2 \delta, 2 \delta) \geq q^{2 \delta(\delta+1)}+\left(q^{2 \delta}-1\right)\left[\begin{array}{c}2 \delta \\ \delta\end{array}\right]_{q}+q^{l}+q^{\delta}+1$, where

$$
l=\left\{\begin{array}{l}
2, \quad \text { if } \delta=1 ; \\
\left(\left\lfloor\frac{\delta}{2}\right\rfloor+1\right) \delta, \quad \text { if } \delta \geq 2 .
\end{array}\right.
$$

Proof By Theorems 2.4 and $2.2, A_{q}^{R}(2 \delta \times 2 \delta, \delta,[0, \delta]) \geq\left(q^{2 \delta}-1\right)\left[\begin{array}{c}2 \delta \\ \delta\end{array}\right]_{q}+1$. Apply Construction 3.17 with $n=4 \delta$ and $k=2 \delta$. Then $l_{1}=2 \delta, l_{2}=\left(\left\lfloor\frac{\delta}{2}\right\rfloor+1\right) \delta$, and so $\max \left\{l_{1}, l_{2}\right\}=l$. 
Combining Corollary 3.18 and Theorem $1.2(3)$ with $n=4 \delta$ and $k=2 \delta$, we have the following corollary.

Corollary 3.19 Let $\delta \geq 2$. Then

$q^{2 \delta(\delta+1)}+\left(q^{2 \delta}-1\right)\left[\begin{array}{c}2 \delta \\ \delta\end{array}\right]_{q}+q^{\left(\left\lfloor\frac{\delta}{2}\right\rfloor+1\right) \delta}+q^{\delta}+1 \leq \bar{A}_{q}(4 \delta, 2 \delta, 2 \delta) \leq q^{2 \delta(\delta+1)}+\left(q^{2 \delta}+q^{\delta}\right)\left[\begin{array}{c}2 \delta \\ \delta\end{array}\right]_{q}+1$.

Remark 3.20 We can calculate the ratio between the lower bound and the upper bound of the CDCs in Corollary 3.19:

$$
\begin{aligned}
\frac{\text { the lower bound of } \mathcal{C}}{\text { the upper bound of } \mathcal{C}} & =\frac{q^{2 \delta(\delta+1)}+\left(q^{2 \delta}-1\right)\left[\begin{array}{c}
2 \delta \\
\delta
\end{array}\right]_{q}+q^{\left(\left\lfloor\frac{\delta}{2}\right\rfloor+1\right) \delta}+q^{\delta}+1}{q^{2 \delta(\delta+1)}+\left(q^{2 \delta}+q^{\delta}\right)\left[\begin{array}{c}
2 \delta \\
\delta
\end{array}\right]_{q}+1} \\
& =1-\frac{\left(q^{\delta}+1\right)\left[\begin{array}{c}
2 \delta \\
\delta
\end{array}\right]_{q}-q^{\left(\left\lfloor\frac{\delta}{2}\right\rfloor+1\right) \delta}-q^{\delta}}{q^{2 \delta(\delta+1)}+\left(q^{2 \delta}+q^{\delta}\right)\left[\begin{array}{c}
2 \delta \\
\delta
\end{array}\right]_{q}+1} \geq \frac{4642}{4797}>0.967688 .
\end{aligned}
$$

Furthermore, for $\delta \geq 3$,

$$
\frac{\text { the lower bound of } \mathcal{C}}{\text { the upper bound of } \mathcal{C}} \geq \frac{16865174}{16877657}>0.99926 \text {. }
$$

To ensure smooth reading of the paper, we move the proof to Appendix A.

There is no systematic approach so far to give a $(4 \delta, 2 \delta, 2 \delta)_{q}$-CDC attaining the lower bound in Corollary 3.19 for general $\delta$. In principle, people can always pick up suitable identifying vectors and then use the multilevel construction to construct an optimal $(4 \delta, 2 \delta, 2 \delta)_{q}$-CDC. However, how to choose identifying vectors effectively is still an open and different problem. The combination of the parallel construction and the multilevel construction helps to weaken the requirement for identifying vectors and provides good constant dimension codes with large size.

\section{Concluding remarks}

Constructions 3.10 and 3.17 for CDCs are established in this paper by combining the parallel construction and the multilevel construction. How to choose identifying vectors compatible with a given parallel construction as many as possible is still an open problem. This paper initials the study.

GRMCs play a fundamental role in our constructions. It is meaningful to investigate various constructions for GRMCs as an independent research topic.

\section{A Appendix}

Proof of Remark 3.20 Write 


$$
f(\delta)=\frac{\left(q^{\delta}+1\right)\left[\begin{array}{c}
2 \delta \\
\delta
\end{array}\right]_{q}-q^{\left(\left\lfloor\frac{\delta}{2}\right\rfloor+1\right) \delta}-q^{\delta}}{q^{2 \delta(\delta+1)}+\left(q^{2 \delta}+q^{\delta}\right)\left[\begin{array}{c}
2 \delta \\
\delta
\end{array}\right]_{q}+1} .
$$

We claim that given any prime power $q, f(\delta)$ is a non-increasing function on $\delta$ for any $\delta \geq 2$. We have

$$
\begin{aligned}
f(\delta)- & f(\delta+1) \\
= & \frac{\left(\left(q^{\delta}+1\right)\left[\begin{array}{c}
2 \delta \\
\delta
\end{array}\right]_{q}-q^{\left(\left\lfloor\frac{\delta}{2}\right\rfloor+1\right) \delta}-q^{\delta}\right)\left(q^{2(\delta+1)(\delta+2)}+\left(q^{2 \delta+2}+q^{\delta+1}\right)\left[\begin{array}{c}
2 \delta+2 \\
\delta+1
\end{array}\right]_{q}+1\right)}{\left(q^{2(\delta+1)(\delta+2)}+\left(q^{2 \delta+2}+q^{\delta+1}\right)\left[\begin{array}{c}
2 \delta+2 \\
\delta+1
\end{array}\right]_{q}+1\right)\left(q^{2 \delta(\delta+1)}+\left(q^{2 \delta}+q^{\delta}\right)\left[\begin{array}{c}
2 \delta \\
\delta
\end{array}\right]_{q}+1\right)} \\
- & \frac{\left(\left(q^{\delta+1}+1\right)\left[\begin{array}{c}
2 \delta+2 \\
\delta+1
\end{array}\right]_{q}-q^{\left(\left\lfloor\frac{\delta+1}{2}\right\rfloor+1\right)(\delta+1)}-q^{\delta+1}\right)\left(q^{2 \delta(\delta+1)}+\left(q^{2 \delta}+q^{\delta}\right)\left[\begin{array}{c}
2 \delta \\
\delta
\end{array}\right]+1\right)}{\left(q_{q}^{2(\delta+1)(\delta+2)}+\left(q^{2 \delta+2}+q^{\delta+1}\right)\left[\begin{array}{c}
2 \delta+2 \\
\delta+1
\end{array}\right]_{q}+1\right)\left(q^{2 \delta(\delta+1)}+\left(q^{2 \delta}+q^{\delta}\right)\left[\begin{array}{c}
2 \delta \\
\delta
\end{array}\right]+1\right)} .
\end{aligned}
$$

Let

$$
\begin{aligned}
g(\delta) & =\left(\left(q^{\delta}+1\right)\left[\begin{array}{c}
2 \delta \\
\delta
\end{array}\right]_{q}-q^{\left(\left\lfloor\frac{\delta}{2}\right\rfloor+1\right) \delta}-q^{\delta}\right)\left(q^{2(\delta+1)(\delta+2)}+\left(q^{2 \delta+2}+q^{\delta+1}\right)\left[\begin{array}{c}
2 \delta+2 \\
\delta+1
\end{array}\right]_{q}+1\right) \\
& -\left(\left(q^{\delta+1}+1\right)\left[\begin{array}{c}
2 \delta+2 \\
\delta+1
\end{array}\right]_{q}-q^{\left(\left\lfloor\frac{\delta+1}{2}\right\rfloor+1\right)(\delta+1)}-q^{\delta+1}\right)\left(q^{2 \delta(\delta+1)}+\left(q^{2 \delta}+q^{\delta}\right)\left[\begin{array}{c}
2 \delta \\
\delta
\end{array}\right]_{q}+1\right) .
\end{aligned}
$$

It suffices to verify $g(\delta) \geq 0$ for any $\delta \geq 2$.

Since $q^{\delta+j}-1 \geq\left(q^{j}-1\right) q^{\delta}$, when $\delta \geq 3$, we have $\left[\begin{array}{c}2 \delta \\ \delta\end{array}\right]_{q}=\frac{\left(q^{2 \delta}-1\right) \cdots\left(q^{\delta+1}-1\right)}{\left(q^{\delta}-1\right) \cdots(q-1)} \geq q^{\delta^{2}} \geq$ $q^{\left(\left\lfloor\frac{\delta}{2}\right\rfloor+1\right) \delta}+q^{\delta}$. When $\delta=2,\left[\begin{array}{l}4 \\ 2\end{array}\right]_{q}=q^{4}+q^{3}+2 q^{2}+q+1>q^{\left(\left\lfloor\frac{2}{2}\right\rfloor+1\right) 2}+q^{2}$. So $\left[\begin{array}{c}2 \delta \\ \delta\end{array}\right]_{q} \geq$ $q^{\left(\left\lfloor\frac{\delta}{2}\right\rfloor+1\right) \delta}+q^{\delta}$ for any $\delta \geq 2$. It follows that

$$
\begin{aligned}
g(\delta) \geq q^{\delta}\left[\begin{array}{c}
2 \delta \\
\delta
\end{array}\right]_{q}\left(q^{2(\delta+1)(\delta+2)}+\left(q^{2 \delta+2}+q^{\delta+1}\right)\left[\begin{array}{c}
2 \delta+2 \\
\delta+1
\end{array}\right]_{q}\right)-\left(\left(q^{\delta+1}+1\right)\left[\begin{array}{c}
2 \delta+2 \\
\delta+1
\end{array}\right]_{q}\right) \times \\
\left(q^{2 \delta(\delta+1)}+\left(q^{2 \delta}+q^{\delta}\right)\left[\begin{array}{c}
2 \delta \\
\delta
\end{array}\right]_{q}+1\right) .
\end{aligned}
$$

Write

$$
g_{1}(\delta)=q^{\delta}\left[\begin{array}{c}
2 \delta \\
\delta
\end{array}\right]_{q}\left(q^{2 \delta+2}+q^{\delta+1}\right)\left[\begin{array}{c}
2 \delta+2 \\
\delta+1
\end{array}\right]_{q}-\left(q^{\delta+1}+1\right)\left[\begin{array}{c}
2 \delta+2 \\
\delta+1
\end{array}\right]_{q}\left(q^{2 \delta}+q^{\delta}\right)\left[\begin{array}{c}
2 \delta \\
\delta
\end{array}\right]_{q}
$$

and

$$
g_{2}(\delta)=q^{\delta}\left[\begin{array}{c}
2 \delta \\
\delta
\end{array}\right]_{q} q^{2(\delta+1)(\delta+2)}-\left(q^{\delta+1}+1\right)\left[\begin{array}{c}
2 \delta+2 \\
\delta+1
\end{array}\right]_{q}\left(q^{2 \delta(\delta+1)}+1\right) .
$$


Then $g(\delta) \geq g_{1}(\delta)+g_{2}(\delta)$. Clearly

$$
g_{1}(\delta)=\left[\begin{array}{c}
2 \delta \\
\delta
\end{array}\right]_{q}\left[\begin{array}{c}
2 \delta+2 \\
\delta+1
\end{array}\right]_{q}\left(q^{3 \delta+2}-q^{3 \delta+1}-q^{2 \delta}-q^{\delta}\right) \geq 0 .
$$

It remains to examine $g_{2}(\delta) \geq 0$. Since $\left[\begin{array}{c}2 \delta+2 \\ \delta+1\end{array}\right]_{q}=\frac{\left(q^{2 \delta+2}-1\right)\left(q^{2 \delta+1}-1\right)}{\left(q^{\delta+1}-1\right)^{2}}\left[\begin{array}{c}2 \delta \\ \delta\end{array}\right]_{q}$ and

$$
q^{\delta}\left(q^{\delta+1}-1\right) \leq q^{2 \delta+1}-1 \leq\left(q^{\delta}+1\right)\left(q^{\delta+1}-1\right),
$$

we have $q^{\delta}\left(q^{\delta+1}+1\right)\left[\begin{array}{c}2 \delta \\ \delta\end{array}\right]_{q} \leq\left[\begin{array}{c}2 \delta+2 \\ \delta+1\end{array}\right]_{q} \leq\left(q^{\delta}+1\right)\left(q^{\delta+1}+1\right)\left[\begin{array}{c}2 \delta \\ \delta\end{array}\right]_{q}$. Thus

$$
\begin{aligned}
g_{2}(\delta) \geq & q^{\delta}\left[\begin{array}{c}
2 \delta \\
\delta
\end{array}\right]_{q} q^{2(\delta+1)(\delta+2)}-\left(q^{\delta+1}+1\right)\left(q^{\delta}+1\right)\left(q^{\delta+1}+1\right)\left[\begin{array}{c}
2 \delta \\
\delta
\end{array}\right]_{q}\left(q^{2 \delta(\delta+1)}+1\right) \\
= & {\left[\begin{array}{c}
2 \delta \\
\delta
\end{array}\right]_{q}\left(q^{2 \delta^{2}+7 \delta+4}-q^{2 \delta^{2}+5 \delta+2}-q^{2 \delta^{2}+4 \delta+2}-2 q^{2 \delta^{2}+4 \delta+1}-2 q^{2 \delta^{2}+3 \delta+1}-q^{2 \delta^{2}+3 \delta}\right.} \\
& \left.-q^{2 \delta^{2}+2 \delta}-q^{3 \delta+2}-q^{2 \delta+2}-2 q^{2 \delta+1}-2 q^{\delta+1}-q^{\delta}-1\right) \geq 0 .
\end{aligned}
$$

So given any prime power $q, f(\delta)$ is a non-increasing function on $\delta$ for any $\delta \geq 2$.

Since

$$
\begin{aligned}
f(2) & =\frac{\left(q^{2}+1\right)\left[\begin{array}{l}
4 \\
2
\end{array}\right]_{q}-q^{4}-q^{2}}{q^{12}+\left(q^{4}+q^{2}\right)\left[\begin{array}{l}
4 \\
2
\end{array}\right]_{q}+1}=\frac{\left(q^{2}+1\right)\left(q^{4}+q^{3}+q^{2}+q+1\right)}{q^{12}+\left(q^{4}+q^{2}\right)\left(q^{2}+1\right)\left(q^{2}+q+1\right)+1} \\
& =\frac{q^{6}+q^{5}+2 q^{4}+2 q^{3}+2 q^{2}+q+1}{q^{12}+q^{8}+q^{7}+3 q^{6}+2 q^{5}+3 q^{4}+q^{3}+q^{2}+1} \leq \frac{155}{4797},
\end{aligned}
$$

we have for any $\delta \geq 2$,

$$
\frac{\text { the lower bound of } \mathcal{C}}{\text { the upper bound of } \mathcal{C}}=1-f(\delta) \geq 1-f(2) \geq 1-\frac{155}{4797}>0.967688 \text {. }
$$

Similarly since

$$
f(3)=\frac{\left(q^{3}+1\right)\left[\begin{array}{l}
6 \\
3
\end{array}\right]_{q}-q^{6}-q^{3}}{q^{24}+\left(q^{6}+q^{3}\right)\left[\begin{array}{l}
6 \\
3
\end{array}\right]_{q}+1} \leq \frac{12483}{16877657},
$$

we have for any $\delta \geq 3$,

$$
\frac{\text { the lower bound of } \mathcal{C}}{\text { the upper bound of } \mathcal{C}}=1-f(\delta) \geq 1-f(3) \geq 1-\frac{12483}{16877657}>0.99926 \text {. }
$$




\section{B Appendix}

Table 1: Constant dimension codes from Theorem 3.12 and 16 Lower bounds for $\bar{A}_{q}(n, 2 \delta, k)$

\begin{tabular}{|c|c|c|}
\hline$\overline{A_{q}(n, 2 \delta, k)}$ & Theorem 3.12 & {$[16]$} \\
\hline$A_{3}(15,6,6)$ & 150102543990846750 & 150102261281924288 \\
\hline$\overline{A_{4}(15,6,6)}$ & 4722384778841908199452 & 4722384497336874172416 \\
\hline$\overline{A_{5}(15,6,6)}$ & 14551922738557090682988320 & 14551922678951263378341888 \\
\hline$\overline{A_{7}(15,6,6)}$ & $\begin{array}{c}2651730911763599010817616 \\
918746\end{array}$ & $\begin{array}{c}2651730911572017468075166 \\
138368\end{array}$ \\
\hline$\overline{A_{8}(15,6,6)}$ & $\begin{array}{c}3245185560810007534450532 \\
05203320\end{array}$ & $\begin{array}{c}3245185560762783660124143 \\
69988608\end{array}$ \\
\hline $\bar{A}_{9}(15,6,6)$ & $\begin{array}{c}2252839960316867802912978 \\
0303636252\end{array}$ & $\begin{array}{c}2252839960308891273979904 \\
2841640960\end{array}$ \\
\hline$\overline{A_{3}(16,6,6)}$ & 12158306011246213950 & 12158283163835867136 \\
\hline $\bar{A}_{4}(16,6,6)$ & 1208930503358636748324892 & 1208930431318239788138496 \\
\hline$\overline{A_{5}(16,6,6)}$ & $\begin{array}{c}909495171159508342705798 \\
8320\end{array}$ & $\begin{array}{c}909495167434454027503612 \\
7232\end{array}$ \\
\hline $\bar{A}_{7}(16,6,6)$ & $\begin{array}{c}6366805919144396570740848 \\
233189146\end{array}$ & $\begin{array}{c}6366805918684414419355934 \\
306336768\end{array}$ \\
\hline$\overline{A_{8}(16,6,6)}$ & $\begin{array}{c}1329228005707779000468281 \\
480881260920\end{array}$ & $\begin{array}{c}1329228005688436187186849 \\
259473338368\end{array}$ \\
\hline $\bar{A}_{9}(16,6,6)$ & $\begin{array}{c}1478088297963896954275969 \\
56677838811932\end{array}$ & $\begin{array}{c}1478088297958663518971939 \\
31748755374080\end{array}$ \\
\hline$\overline{A_{3}(17,6,6)}$ & 984822786754900790910 & 984820936270705197056 \\
\hline$\overline{A_{4}(17,6,6)}$ & 309486208859711440279978012 & 309486190417469385763454976 \\
\hline $\bar{A}_{5}(17,6,6)$ & $\begin{array}{c}56843448197469116506558079 \\
88320\end{array}$ & $\begin{array}{c}56843447964653369704091609 \\
98912\end{array}$ \\
\hline$\overline{A_{7}(17,6,6)}$ & $\begin{array}{c}15286701011865696133769150 \\
164214433946\end{array}$ & $\begin{array}{c}15286701010761278864076273 \\
642983391232\end{array}$ \\
\hline$\overline{A_{8}(17,6,6)}$ & $\begin{array}{c}54445179113790627852329396 \\
89634331511160\end{array}$ & $\begin{array}{c}54445179112998346227173345 \\
66802793955328\end{array}$ \\
\hline$\overline{A_{9}}(17,6,6)$ & $\begin{array}{c}96977373229411279169036992 \\
0953064514284572\end{array}$ & $\begin{array}{c}96977373229067909497849520 \\
2646735567454208\end{array}$ \\
\hline $\bar{A}_{3}(17,6,7)$ & 717934513945606807214448 & 717934462541344066764800 \\
\hline$\overline{A_{4}(17,6,7)}$ & $\begin{array}{c}1267655437138714999914722 \\
735680\end{array}$ & $\begin{array}{c}1267655435949954604087111 \\
581696\end{array}$ \\
\hline$\overline{A_{5}(17,6,7)}$ & $\begin{array}{c}8881788744768854329316669 \\
4301204500\end{array}$ & $\begin{array}{c}8881788744477090184835827 \\
3727594496\end{array}$ \\
\hline$\overline{A_{7}(17,6,7)}$ & $\begin{array}{c}1798465087215432610771286 \\
951668456912186048\end{array}$ & $\begin{array}{c}1798465087215053471877135 \\
499494277221711872\end{array}$ \\
\hline $\bar{A}_{8}(17,6,7)$ & $\begin{array}{c}1427247703339824504317286 \\
302786023739979637248 \\
\end{array}$ & $\begin{array}{c}1427247703339783847337612 \\
952679951618625503232 \\
\end{array}$ \\
\hline$\overline{A_{9}}(17,6,7)$ & $\begin{array}{c}5153775220622933028375569 \\
74561316380272313825860\end{array}$ & $\begin{array}{c}5153775220622908412230038 \\
58973884622428476276736\end{array}$ \\
\hline $\bar{A}_{3}(18,6,7)$ & 174458086133950569694999932 & 174458074397546630638206976 \\
\hline
\end{tabular}


Table 1 (Cont.): Constant dimension codes from Theorem 3.12 and [16] Lower bounds for $\bar{A}_{q}(n, 2 \delta, k)$

\begin{tabular}{|c|c|c|}
\hline $\bar{A}_{q}(n, 2 \delta, k)$ & Theorem [3.12 & {$[16]$} \\
\hline $\bar{A}_{4}(18,6,7)$ & 129807916760321574216291241 & 129807916641275351458520225 \\
& 3988416 & 9656704 \\
\hline $\bar{A}_{5}(18,6,7)$ & 277555898273931997862551572 & 277555898264909066117850548 \\
& 409926204500 & 774715260928 \\
\hline $\bar{A}_{7}(18,6,7)$ & 302268027208297537838299541 & 302268027208234077195734138 \\
& 69875398154049929084 & 40701999450419101696 \\
\hline $\bar{A}_{8}(18,6,7)$ & 467680527430393663434529587 & 467680527430380371095589012 \\
& 83305087356415231304192 & 33416654639120489906176 \\
\hline $\bar{A}_{9}(18,6,7)$ & 304325273002563570083080541 & 304325273002562128138529778 \\
& 77589445454500732900196836 & 45672945438783195662254080 \\
\hline$A_{3}(19,6,7)$ & 42393314923753431509633199 & 42393312078603826847037784 \\
& 312 & 064 \\
\hline$A_{4}(19,6,7)$ & 132923306762526363919145683 & 132923306640665959893524711 \\
& 3419327040 & 3888464896 \\
\hline$A_{5}(19,6,7)$ & 867362182106035125792002834 & 867362182077840679822534739 \\
& 689855238704500 & 559109441355776 \\
\hline $\bar{A}_{7}(19,6,7)$ & 508021873328985670761663974 & 508021873328878943122344222 \\
& 813537143982278408961152 & 542078792818337377157120 \\
\hline$A_{8}(19,6,7)$ & 153249555228391395614936944 & 153249555228387040000602607 \\
& 5210953425471736128141435392 & 5616596939214700213245575168 \\
\hline$A_{9}(19,6,7)$ & 17970103045528376249648755925 & 17970103045528289061322277127 \\
& 65293397244462194049577733380 & 01292762916333299089237082112 \\
\hline
\end{tabular}


Table 2: Constant dimension codes from Theorem 3.12, 3.14, 3.16 and 16] Lower bounds for $\bar{A}_{q}(n, 2 \delta, k)$

\begin{tabular}{|c|c|c|c|c|}
\hline $\bar{A}_{q}(n, 2 \delta, k)$ & Theorem 3.16 & Theorem 3.14 & Theorem 3.12 & 116 \\
\hline$A_{3}(13,4,5)$ & $\begin{array}{c}187977330080 \\
0662\end{array}$ & $\begin{array}{c}187644030023 \\
1043\end{array}$ & $\begin{array}{c}187623140212 \\
3284\end{array}$ & $\begin{array}{c}187646890063 \\
3708\end{array}$ \\
\hline$\overline{A_{4}(13,4,5)}$ & $\begin{array}{c}185244551321 \\
42240085\end{array}$ & $\begin{array}{c}185193502027 \\
94936427\end{array}$ & $\begin{array}{c}185191080330 \\
51471424\end{array}$ & $\begin{array}{c}185193668253 \\
31922597\end{array}$ \\
\hline $\bar{A}_{5}(13,4,5)$ & $\begin{array}{c}233220333417 \\
60498047656\end{array}$ & $\begin{array}{c}233204343725 \\
35344411636\end{array}$ & $\begin{array}{c}233203799712 \\
28104104500\end{array}$ & $\begin{array}{c}233204366687 \\
01425801556\end{array}$ \\
\hline$\overline{A_{7}(13,4,5)}$ & $\begin{array}{c}110489772145 \\
826259551989 \\
4274\end{array}$ & $\begin{array}{c}110488804026 \\
935735689413 \\
6924\end{array}$ & $\begin{array}{c}110488785701 \\
592348093173 \\
0404\end{array}$ & $\begin{array}{c}110488804410 \\
257915997937 \\
0508\end{array}$ \\
\hline$\overline{A_{8}(13,4,5)}$ & $\begin{array}{c}792478237928 \\
289801654459 \\
47977\end{array}$ & $\begin{array}{c}792475147017 \\
480054665841 \\
57579\end{array}$ & $\begin{array}{c}792475101353 \\
872707790607 \\
24224\end{array}$ & $\begin{array}{c}792475147744 \\
175434285903 \\
14633\end{array}$ \\
\hline$\overline{A_{9}(13,4,5)}$ & $\begin{array}{c}343421389872 \\
099066234534 \\
2116660\end{array}$ & $\begin{array}{c}343420732650 \\
983522582815 \\
7580092\end{array}$ & $\begin{array}{c}343420724881 \\
350622237717 \\
6247844\end{array}$ & $\begin{array}{c}343420732748 \\
274503618449 \\
0814932\end{array}$ \\
\hline$\overline{\bar{A}_{3}(14,4,5)}$ & $\begin{array}{c}152290128114 \\
549994\end{array}$ & $\begin{array}{c}151991664671 \\
267113\end{array}$ & $\begin{array}{c}151971000771 \\
313764\end{array}$ & $\begin{array}{c}151993980961 \\
035277\end{array}$ \\
\hline$\overline{A_{4}(14,4,5)}$ & $\begin{array}{c}44234835438 \\
9224194389\end{array}$ & $\begin{array}{c}44095365198 \\
0418663364\end{array}$ & $\begin{array}{c}474088568287 \\
3806466624\end{array}$ & $\begin{array}{c}474095790728 \\
5780822949\end{array}$ \\
\hline$\overline{A_{5}(14,4,5)}$ & $\begin{array}{c}145763149391 \\
911682128914 \\
06\end{array}$ & $\begin{array}{c}145752714828 \\
382638784555 \\
29\end{array}$ & $\begin{array}{c}145752356230 \\
952762603545 \\
00\end{array}$ & $\begin{array}{c}145752729179 \\
384155801334 \\
31\end{array}$ \\
\hline$\overline{A_{7}(14,4,5)}$ & $\begin{array}{c}265285995217 \\
197106992212 \\
2343434\end{array}$ & $\begin{array}{c}265283618468 \\
672861039951 \\
4145353\end{array}$ & $\begin{array}{c}265283573374 \\
849294506738 \\
6763204\end{array}$ & $\begin{array}{c}265283619389 \\
029256718151 \\
6018577\end{array}$ \\
\hline & $\begin{array}{c}324599107938 \\
548775888100 \\
448539209\end{array}$ & $\begin{array}{c}324597820218 \\
359848132646 \\
389432068\end{array}$ & $\begin{array}{c}324597801169 \\
235252976985 \\
412575744\end{array}$ & $\begin{array}{c}324597820516 \\
014257914299 \\
810657417\end{array}$ \\
\hline$\overline{A_{9}(14,4,5)}$ & $\begin{array}{c}225318779695 \\
316687971945 \\
70848710934\end{array}$ & $\begin{array}{c}225318342692 \\
310290649450 \\
77022477769\end{array}$ & $\begin{array}{c}225318337521 \\
979435078382 \\
85688931844\end{array}$ & $\begin{array}{c}225318342756 \\
142901825894 \\
86571731339\end{array}$ \\
\hline
\end{tabular}


Table 3: Constant dimension codes from Construction 3.17 and [16]

Lower bounds for $\bar{A}_{q}(n, 2 \delta, k)$

\begin{tabular}{|c|c|c|}
\hline $\bar{A}_{q}(n, 2 \delta, k)$ & Construction 3.17 & 16 \\
\hline$A_{2}(12,6,6)$ & 16865174 & 16865101 \\
\hline$\overline{A_{3}(12,6,6)}$ & 282454201878 & 282454201122 \\
\hline $\bar{A}_{4}(12,6,6)$ & 281476519731292 & 281476519727132 \\
\hline$\overline{A_{5}(12,6,6)}$ & 59604684750285320 & 59604684750269570 \\
\hline $\bar{A}_{7}(12,6,6)$ & 191581237048517757994 & 191581237048517640002 \\
\hline$\overline{A_{8}(12,6,6)}$ & 4722366523787007642488 & 4722366523787007379832 \\
\hline $\bar{A}_{9}(12,6,6)$ & 79766443311676870585932 & 79766443311676870053762 \\
\hline $\bar{A}_{2}(14,6,7)$ & 34532242376 & 34532238023 \\
\hline$A_{3}(14,6,7)$ & 50035894106925204 & 50035894106387202 \\
\hline $\bar{A}_{4}(14,6,7)$ & 1180598085852258350656 & 1180598085852241507904 \\
\hline$A_{5}(14,6,7)$ & 2910384996920980879329500 & 2910384996920980634798250 \\
\hline$\overline{A_{7}(14,6,7)}$ & 378818703472375564731912769036 & 378818703472375564718065717034 \\
\hline $\bar{A}_{8}(14,6,7)$ & $\begin{array}{c}40564819558769908757756030657 \\
024\end{array}$ & $\begin{array}{c}40564819558769908757687294403 \\
072\end{array}$ \\
\hline $\bar{A}_{9}(14,6,7)$ & $\begin{array}{c}25031555123615248786076588088 \\
37716\end{array}$ & $\begin{array}{c}25031555123615248786073763362 \\
54514\end{array}$ \\
\hline$\overline{A_{2}(16,8,8)}$ & 1099562832574 & 1099562828461 \\
\hline$A_{3}(16,8,8)$ & 12157665957048196644 & 12157665957047665122 \\
\hline $\bar{A}_{4}(16,8,8)$ & 1208925820022362634893084 & 1208925820022362618115612 \\
\hline$A_{5}(16,8,8)$ & 9094947017807612368246590820 & 9094947017807612368002449570 \\
\hline $\bar{A}_{7}(16,8,8)$ & $\begin{array}{c}63668057609092569002476703621 \\
23204\end{array}$ & $\begin{array}{c}63668057609092569002476565208 \\
33602\end{array}$ \\
\hline$\overline{A_{8}(16,8,8)}$ & $\begin{array}{c}13292279957849213674394204065 \\
92780664\end{array}$ & $\begin{array}{c}13292279957849213674394203378 \\
73299832\end{array}$ \\
\hline $\bar{A}_{9}(16,8,8)$ & $\begin{array}{c}14780882941434601431198749325 \\
1158647364\end{array}$ & $\begin{array}{c}14780882941434601431198749296 \\
8729104322\end{array}$ \\
\hline$\overline{A_{2}(16,6,8)}$ & 282927684131264 & 282927683836351 \\
\hline$\overline{A_{3}(16,6,8)}$ & 79773403858211769073398 & 79773403858211367304002 \\
\hline$\overline{A_{4}(16,6,8)}$ & 79228596795209597355803963392 & 79228596795209597286010744832 \\
\hline$\overline{A_{5}(16,6,8)}$ & $\begin{array}{c}3552716061446350478567982091 \\
625000\end{array}$ & $\begin{array}{c}3552716061446350478564136876 \\
781250\end{array}$ \\
\hline$\overline{A_{7}(16,6,8)}$ & $\begin{array}{c}3670336930316550640268162626 \\
0312448748394\end{array}$ & $\begin{array}{c}3670336930316550640268162462 \\
7151289328002\end{array}$ \\
\hline$\overline{A_{8}(16,6,8)}$ & $\begin{array}{c}2230074539175728767236156261 \\
8047925701050368\end{array}$ & $\begin{array}{c}2230074539175728767236156259 \\
9998342819479552\end{array}$ \\
\hline $\bar{A}_{9}(16,6,8)$ & $\begin{array}{c}6362685459865446204861526038 \\
705059941329515532\end{array}$ & $\begin{array}{c}63626854598654462048615260385 \\
54759414900421762\end{array}$ \\
\hline $\bar{A}_{2}(18,8,9)$ & 18015215398134856 & 18015215398068295 \\
\hline $\bar{A}_{3}(18,8,9)$ & 58149739380417667241629716 & 58149739380417667198523946 \\
\hline$\overline{A_{4}(18,8,9)}$ & $\begin{array}{c}32451855376784298642321718 \\
2266944\end{array}$ & $\begin{array}{c}32451855376784298642321288 \\
6251072\end{array}$ \\
\hline $\bar{A}_{5}(18,8,9)$ & $\begin{array}{c}55511151231735878357960116 \\
981761704500\end{array}$ & $\begin{array}{c}55511151231735878357960116 \\
829164048250\end{array}$ \\
\hline
\end{tabular}


Table 3 (Cont.): Constant dimension codes from Construction 3.17 and [16 Lower bounds for $\bar{A}_{q}(n, 2 \delta, k)$

\begin{tabular}{|c|c|c|}
\hline $\bar{A}_{q}(n, 2 \delta, k)$ & Construction $[3.17$ & {$[16]$} \\
\hline$A_{7}(18,8,9)$ & 43181145673965918176230160 & 43181145673965918176230160 \\
& 95285332497749370596 & 95285299264536325746 \\
\hline $\bar{A}_{8}(18,8,9)$ & 58460065493236358379340343 & 58460065493236358379340343 \\
& 02923933871658428965376 & 02923933590182378512896 \\
\hline $\bar{A}_{9}(18,8,9)$ & 33813919135227284246202802 & 33813919135227284246202802 \\
& 47018514715266280331502884 & 47018514713413256655866642 \\
\hline $\bar{A}_{2}(18,6,9)$ & 9271545156585415680 & 9271545156551861247 \\
\hline$A_{3}(18,6,9)$ & 11446612801881132293137038 & 11446612801881132287488447 \\
& 59802 & 86840 \\
\hline$A_{4}(18,6,9)$ & 85071058146182803276503914 & 85071058146182803276503351 \\
& 069802090496 & 119848669184 \\
\hline $\bar{A}_{5}(18,6,9)$ & 10842028996571097790669084 & 108420289965710977906690845 \\
& 5136306309921875000 & 017097020371093750 \\
\hline $\bar{A}_{7}(18,6,9)$ & 174251503388975551318884922 & 174251503388975551318884922 \\
& 599369849935281754612330830 & 599369466772818993479502028 \\
\hline $\bar{A}_{8}(18,6,9)$ & 78463772372191979113838163463 & 78463772372191979113838163463 \\
& 5235743275201736965558894592 & 5235733830468771226268467200 \\
\hline$A_{9}(18,6,9)$ & 1310020512493866339206870302329 & 1310020512493866339206870302329 \\
& 188713507904303585133560754636 & 188713348371417431388541027914 \\
\hline
\end{tabular}

\section{References}

[1] R. Ahlswede and H. Aydinian, On error control codes for random network coding, in Proc. Workshop Netw. Coding Theory Appl., 2009, 68-73.

[2] J. Antrobus and H. Gluesing-Luerssen, Maximal Ferrers diagram codes: constructions and genericity considerations, IEEE Trans. Inf. Theory, 65 (2019), 6204-6223.

[3] H. Chen, X. He, J. Weng, and L. Xu, New constructions of subspace codes using subsets of MRD codes in several blocks, arXiv:1908.03804 v1.

[4] P. Delsarte, Bilinear forms over a finite field, with applications to coding theory, $J$. Combin. Theory A, 25 (1978), 226-241.

[5] T. Etzion, E. Gorla, A. Ravagnani and A. Wachter-Zeh, Optimal Ferrers diagram rank-metric codes, IEEE Trans. Inf. Theory, 62 (2016), 1616-1630.

[6] T. Etzion and N. Silberstein, Error-correcting codes in projective spaces via rankmetric codes and Ferrers diagrams, IEEE Trans. Inf. Theory, 55 (2009), 2909-2919.

[7] T. Etzion and N. Silberstein, Codes and designs related to lifted MRD codes, IEEE Trans. Inf. Theory, 59 (2013), 1004-1017.

[8] T. Etzion and A. Vardy, Error-correcting codes in projective spaces, IEEE Trans. Inf. Theory, 57 (2011), 1165-1173. 
[9] P. Frankl and R.M. Wilson, The Erdös-Ko-Rado theorem for vector spaces, J. Combin. Theory A, 43 (1986), 228-236.

[10] È.M. Gabidulin, Theory of codes with maximum rank distance, Problems Inf. Transmiss., 21 (1985), 3-16.

[11] M. Gadouleau and Z. Yan, Constant-rank codes and their connection to constantdimension codes, IEEE Trans. Inform. Theory, 56 (2010), 3207-3216.

[12] H. Gluesing-Luerssen and C. Troha, Construction of subspace codes through linkage, Adv. in Math. of Comm., 10 (2016), 525-540.

[13] E. Gorla and A. Ravagnani, Subspace codes from Ferrers diagrams, J. Algebra and its Appl., 16 (2017), 1750131.

[14] D. Heinlein, New LMRD code bounds for constant dimension codes and improved constructions, IEEE Trans. Inform. Theory, 65 (2019), 4822-4830.

[15] D. Heinlein, Generalized linkage construction for constant-dimension codes, arXiv:1910.11195v2.

[16] D. Heinlein, M. Kiermaier, S. Kurz, and A. Wassermann, Tables of subspace codes, http://subspacecodes.uni-bayreuth.de.

[17] S. Liu, Y. Chang and T. Feng, Constructions for optimal Ferrers diagram rankmetric codes, IEEE Trans. Inf. Theory, 65 (2019), 4115-4130.

[18] S. Liu, Y. Chang and T. Feng, Several classes of optimal Ferrers diagram rank-metric codes, Linear Algebra and its Appl., 581 (2019), 128-144.

[19] A. Kohnert and S. Kurz, Construction of large constant dimension codes with a prescribed minimum distance, Lecture Notes Comp. Sci., 5393 (2008), 31-42.

[20] R. Kötter and F.R. Kschischang, Coding for errors and erasures in random network coding, IEEE Trans. Inf. Theory, 54 (2008), 3579-3591.

[21] R.M. Roth, Maximum-rank array codes and their application to crisscross error correction, IEEE Trans. Inf. Theory, 37 (1991), 328-336.

[22] N. Silberstein and T. Etzion, Large constant dimension codes and lexicodes, Adv. in Math. of Comm., 5 (2011), 177-189.

[23] N. Silberstein and T. Etzion, Enumerative coding for Grassmannian space, IEEE Trans. Inf. Theory, 57 (2011), 365-374.

[24] N. Silberstein and A.-L. Trautmann, Subspace codes based on graph matchings, Ferrers diagrams, and pending blocks, IEEE Trans. Inf. Theory, 61 (2015), 39373953.

[25] D. Silva, F.R. Kschischang, and R. Kötter, A rank-metric approach to error control in random network coding, IEEE Trans. Inf. Theory, 54 (2008), 3951-3967.

[26] V. Skachek, Recursive code construction for random networks, IEEE Trans. Inf. Theory, 56 (2010), 1378-1382. 
[27] A.-L. Trautmann, F. Manganiello, M. Braun, and J. Rosenthal, Cyclic orbit codes, IEEE Trans. Inf. Theory, 59 (2013), 7386-7404.

[28] A.-L. Trautmann and J. Rosenthal, New improvements on the Echelon-Ferrers construction, in Proc. 19th Int. Symp. Math. Theory Netw. Syst., Jul. (2010), 405-408.

[29] H. Wang, C. Xing, and R. Safavi-Naini, Linear authentication codes: bounds and constructions, IEEE Trans. Inf. Theory, 49 (2003), 866-872.

[30] S.-T. Xia and F.-W. Fu, Johnson type bounds on constant dimension codes, Des. Codes Cryptogr., 50 (2009), 163-172.

[31] L. Xu and H. Chen, New constant-dimension subspace codes from maximum rank distance codes, IEEE Trans. Inf. Theory, 64 (2018), 6315-6319.

[32] T. Zhang and G. Ge, Constructions of optimal Ferrers diagram rank metric codes, Des. Codes Cryptogr., 87 (2019), 107-121. 